\title{
Charm spectroscopy and exotics at ZEUS
}

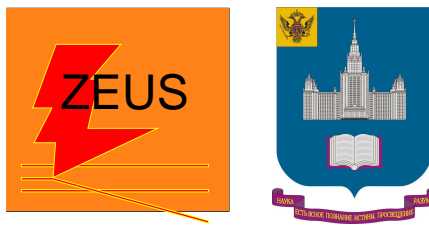

\author{
Leonid Gladilin \\ (SINP MSU)
}

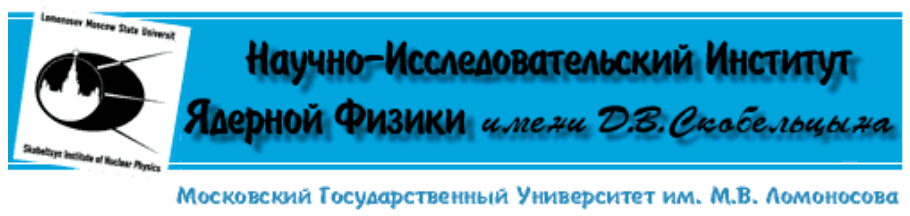

ICHEP 06, 26.07 - 2.08, Moscow

XXXIII INTERNATIONAL CONFERENCE ON HIGH ENERGY PHYSICS

\section{O U T L I N E :}

\section{HERA and HERA II}

results on $\Theta^{+} \rightarrow K_{s}^{0} p(+$ c.c. $)$

$d / \bar{d}$ production in DIS

search for $\Theta_{c}^{0} \rightarrow D^{*-} p(+$ c.c. $)$

excited $D$ mesons

Summary and Outlook

B A C K U P :

more on $\Theta^{+} \rightarrow K_{s}^{0} p$ (+c.c.)

$\Theta^{++}$and $\Xi_{3 / 2}^{--, 0}$ searches

more on $\Theta_{c}^{0} \rightarrow D^{*-} p(+$ c.c. $)$

more on excited $D$ mesons 


\section{HERA}
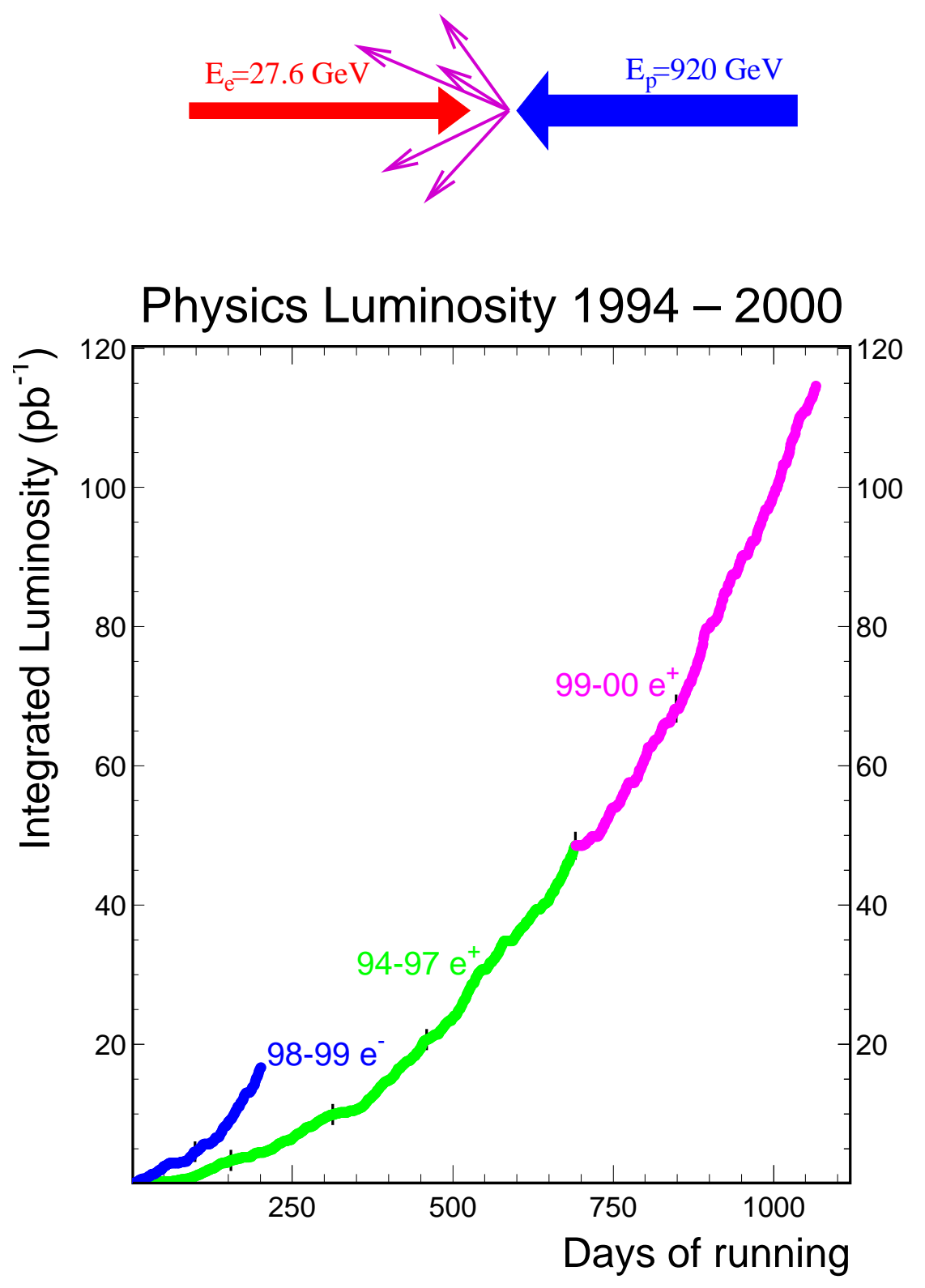

H1, ZEUS : > $100 \mathrm{pb}^{-1}$ each

\section{HERA II}

\section{HERA}

1992-2000

$320(300)$

$1.5 \cdot 10^{31}$

0.1
HERA II

2003-2007

$320 \mathrm{GeV}$

$7 \cdot 10^{31} \mathrm{~cm}^{-2} \mathrm{~s}^{-1}$

$\sim 0.5 \mathrm{fb}^{-1}$

$$
e(k)+p(P) \rightarrow e\left(k^{\prime}\right)+X \quad s=(\boldsymbol{P}+\boldsymbol{k})^{2}
$$

$$
Q^{2}=-q^{2}=-\left(k-k^{\prime}\right)^{2}
$$

Photoproduction $\quad Q^{2} \simeq 0 \mathrm{GeV}^{2}$ DIS $Q^{2}>1 \mathrm{GeV}^{2}$

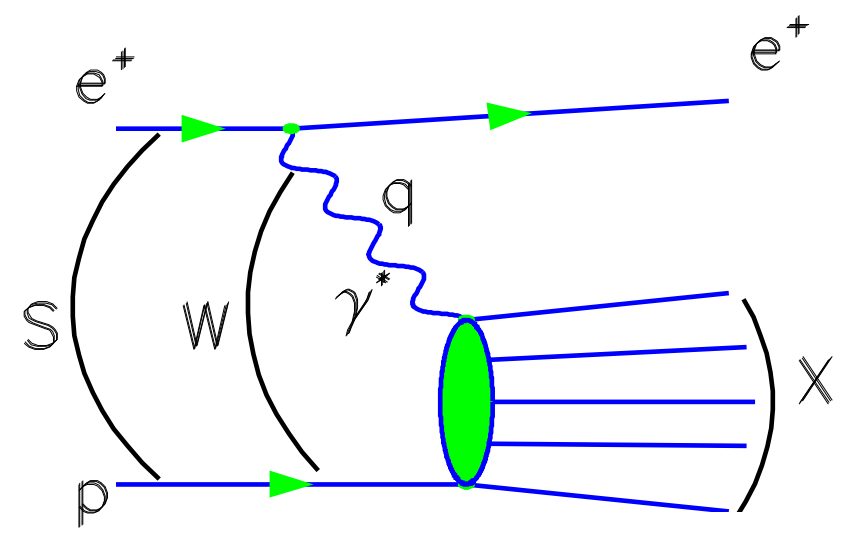

$$
\begin{aligned}
& W^{2}=(P+q)^{2} \\
& y=\frac{q P}{k P} \cong \frac{W^{2}+Q^{2}}{s} \\
& x=\frac{Q^{2}}{2 q P} \cong \frac{Q^{2}}{s y}
\end{aligned}
$$




\section{Strange pentaquarks}

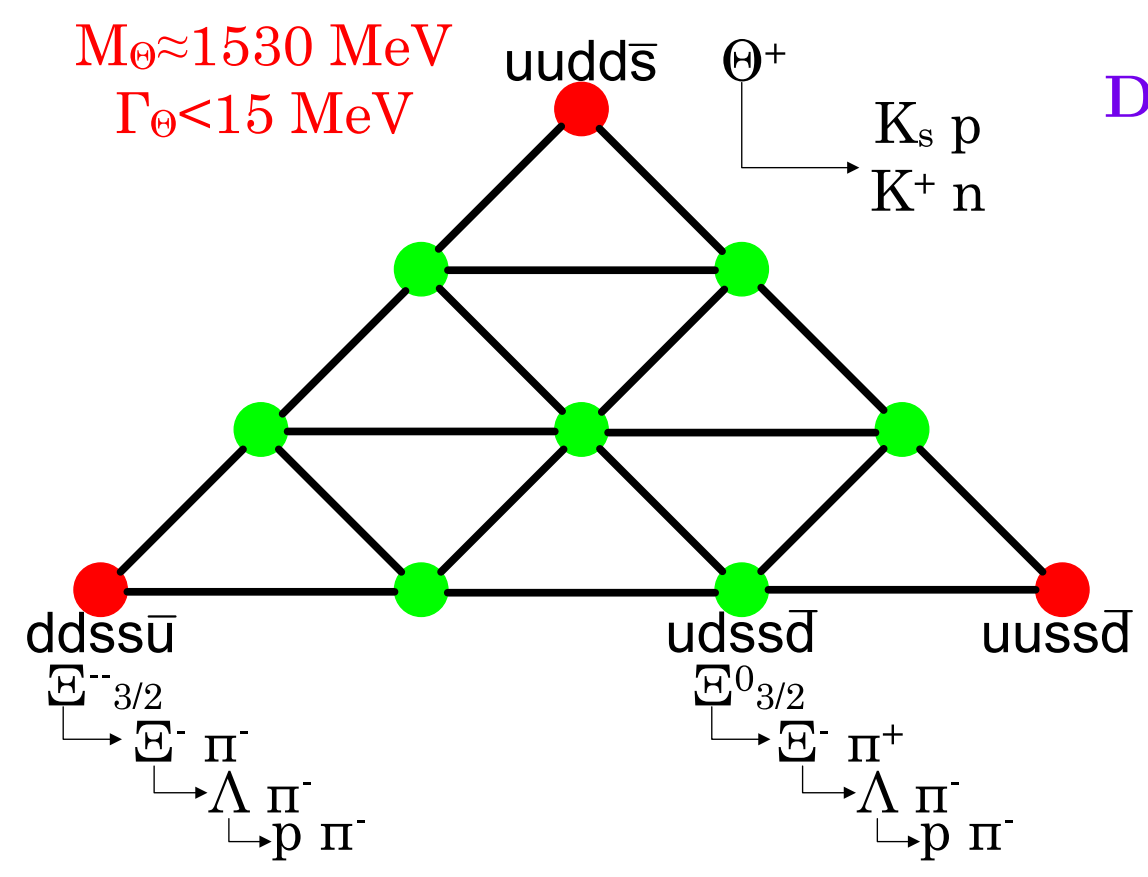

Diakonov, Petrov, Polyakov (hep-ph/9703373)

Exotic Anti-Decuplet of Baryons:

predictions from Chiral Solitons

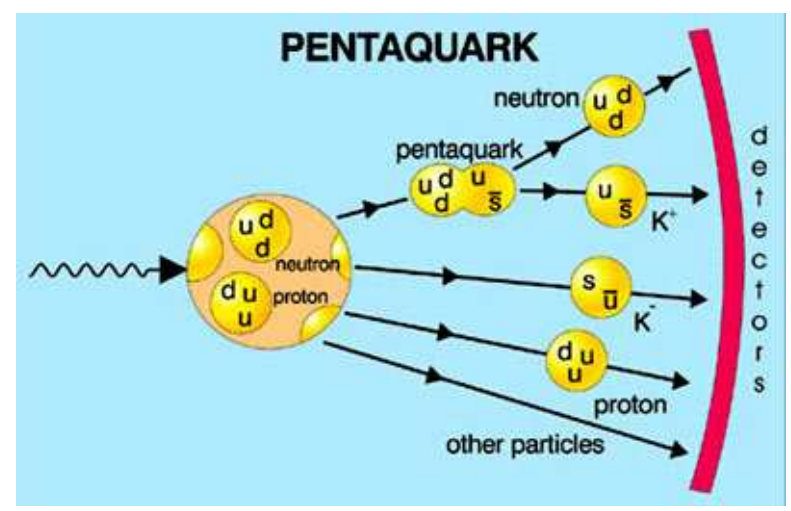

exotic $(\mathrm{S}=\mathrm{B}=+1)$ narrow baryon $\Theta^{+} \rightarrow K^{+} n$ observed by LEPS, CLAS, SAPHIR non-exotic decay mode $\Theta^{+} \rightarrow K_{s}^{0} p$ seen by DIANA, HERMES, COSY-TOF, SVD, ZEUS, ITEP

negative results from BES, HERA-B, CDF, ALEPH, DELPHI, L3, BABAR, BELLE, SPHINX, HyperCP, PHENIX and ... CLAS $\left(\gamma p \rightarrow K_{s}^{0} K^{+} n\right)$ with $50 \times$ SAPHIR

another exotic $(\mathrm{S}=-2, \mathrm{~B}=+1)$ narrow baryon $\Xi_{3 / 2}^{--} \rightarrow \Xi^{-} \pi^{-}$reported by NA49 negative results from WA89, HERA-B, HERMES, CDF, ALEPH, BABAR, ZEUS, $\cdots$ 


\section{$M\left(K_{s}^{0} p(\bar{p})\right)$ for $Q^{2}>20 \mathrm{GeV}^{2}$}

ZEUS

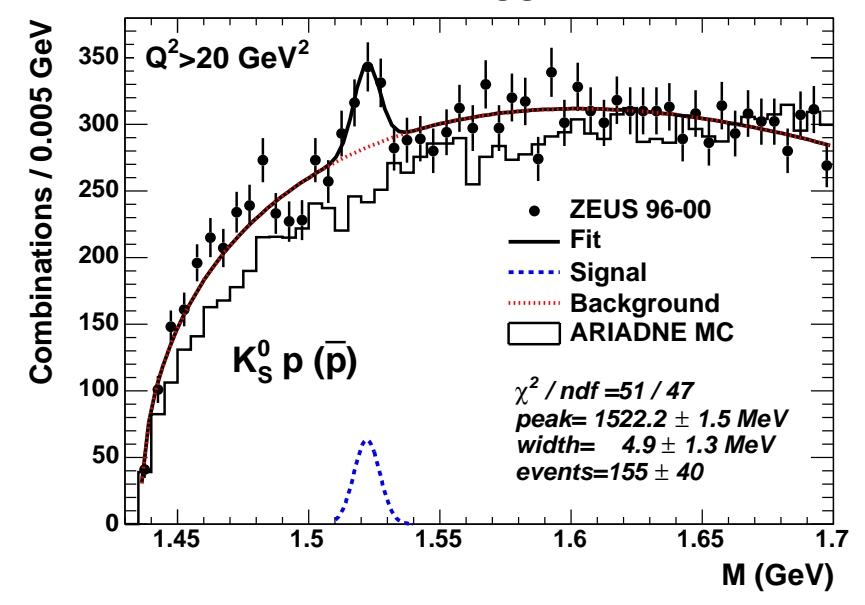

$Q^{2}>20 \mathrm{GeV}^{2}:$ best signal identification

Fit with Gaussian + background (3 par.)

$$
N=155 \pm 40, M=1522.2 \pm 1.5 \mathrm{MeV}
$$

width compatible with resolution

ZEUS

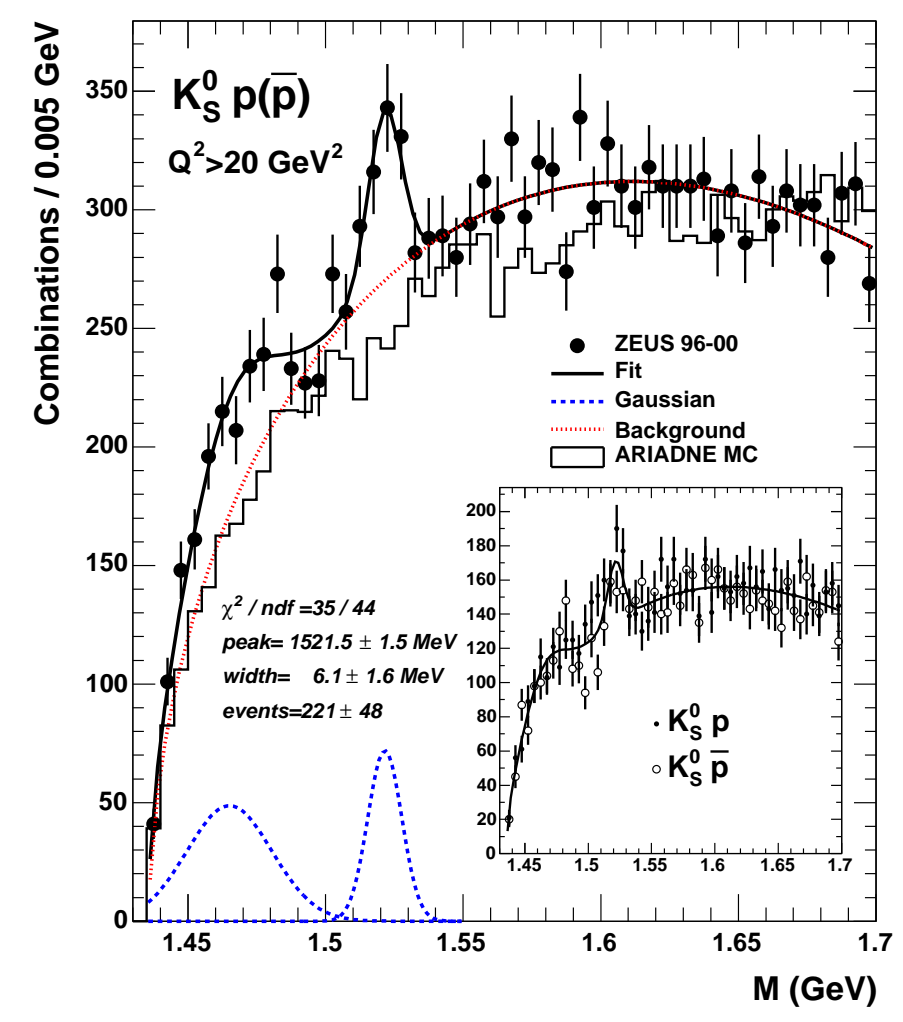

Fit with 2nd Gaussian for ( $\Sigma$ ?) bump around $1465 \mathrm{MeV}$

$$
N=221 \pm 48, M=1521.5 \pm 1.5 \mathrm{MeV}
$$

width compatible with resolution

For BW: $\Gamma=8 \pm 4$ (stat.) $\mathrm{MeV}$

$\Longleftarrow$ signal seen in both charges

$$
N\left(\Theta^{-} \rightarrow K_{s}^{0} \bar{p}\right)=96 \pm 34
$$




\section{$\Theta^{+}$cross section (ZEUS) and upper limit on it $(\mathrm{H} 1)$}

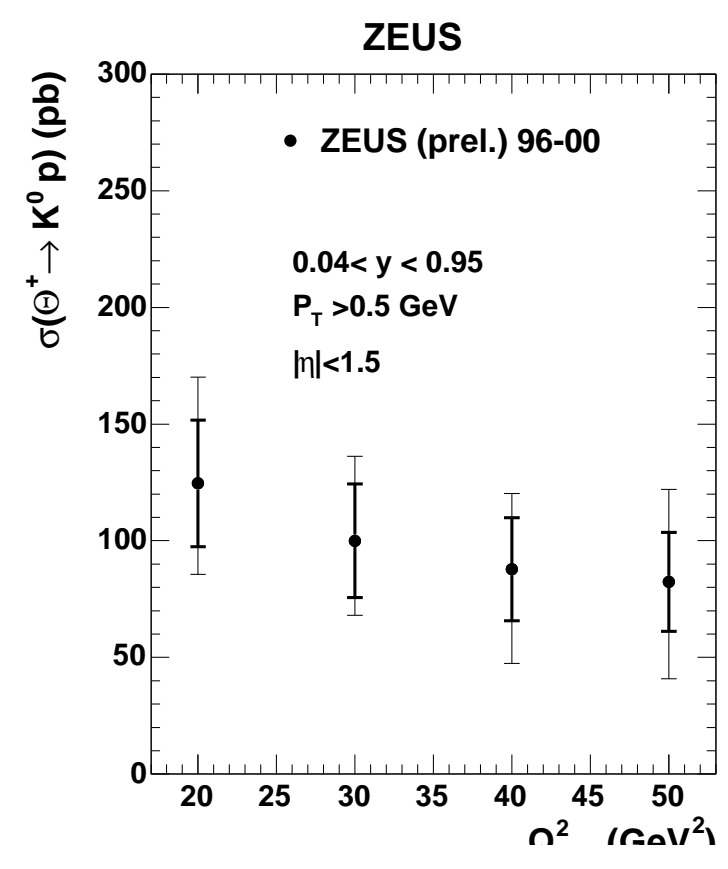

$\Theta^{ \pm}$cross section in the visible range:

$$
\begin{gathered}
Q^{2}>20 \mathrm{GeV}^{2}, 0.04<y<0.95 \\
p_{T}\left(\Theta^{ \pm}\right)>0.5 \mathrm{GeV},\left|\eta\left(\Theta^{ \pm}\right)\right|<1.5 \\
\sigma\left(e p \rightarrow e \Theta^{+} X \rightarrow e K^{0} p X\right)=125 \pm 27_{-28}^{+36} \mathrm{pb} \\
R=\sigma\left(\Theta^{+} \rightarrow K^{0} p\right) / \sigma\left(\Lambda^{0}\right)=4.2 \pm 0.9_{-0.9}^{+1.2} \% \\
\text { HERA-B: } R<0.46 \% \\
\text { ALEPH: } R<0.4 \%
\end{gathered}
$$

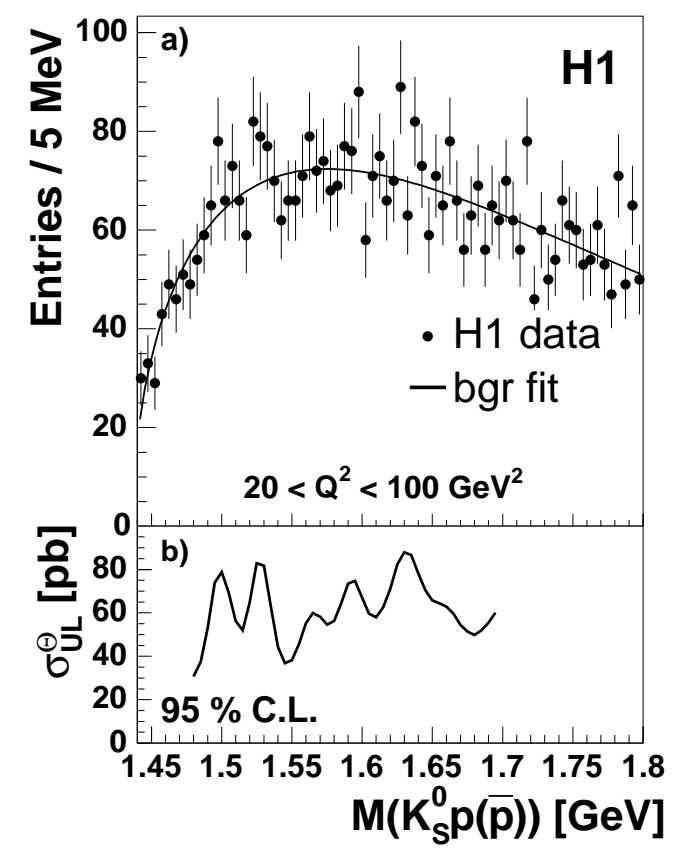

H1 : no significant signal in particular, for $Q^{2}>20 \mathrm{GeV}^{2}$

consistent with the ZEUS measurement

$$
\begin{gathered}
\text { note }: \mathcal{L}_{\text {int }}(\text { ZEUS })=121 \mathrm{pb}^{-1} \\
\mathcal{L}_{\text {int }}(\mathrm{H} 1)=74 \mathrm{pb}^{-1}
\end{gathered}
$$

larger luminosity is vital 


\section{$\Theta^{+}$production mechanism in $e p$ collisions ?}
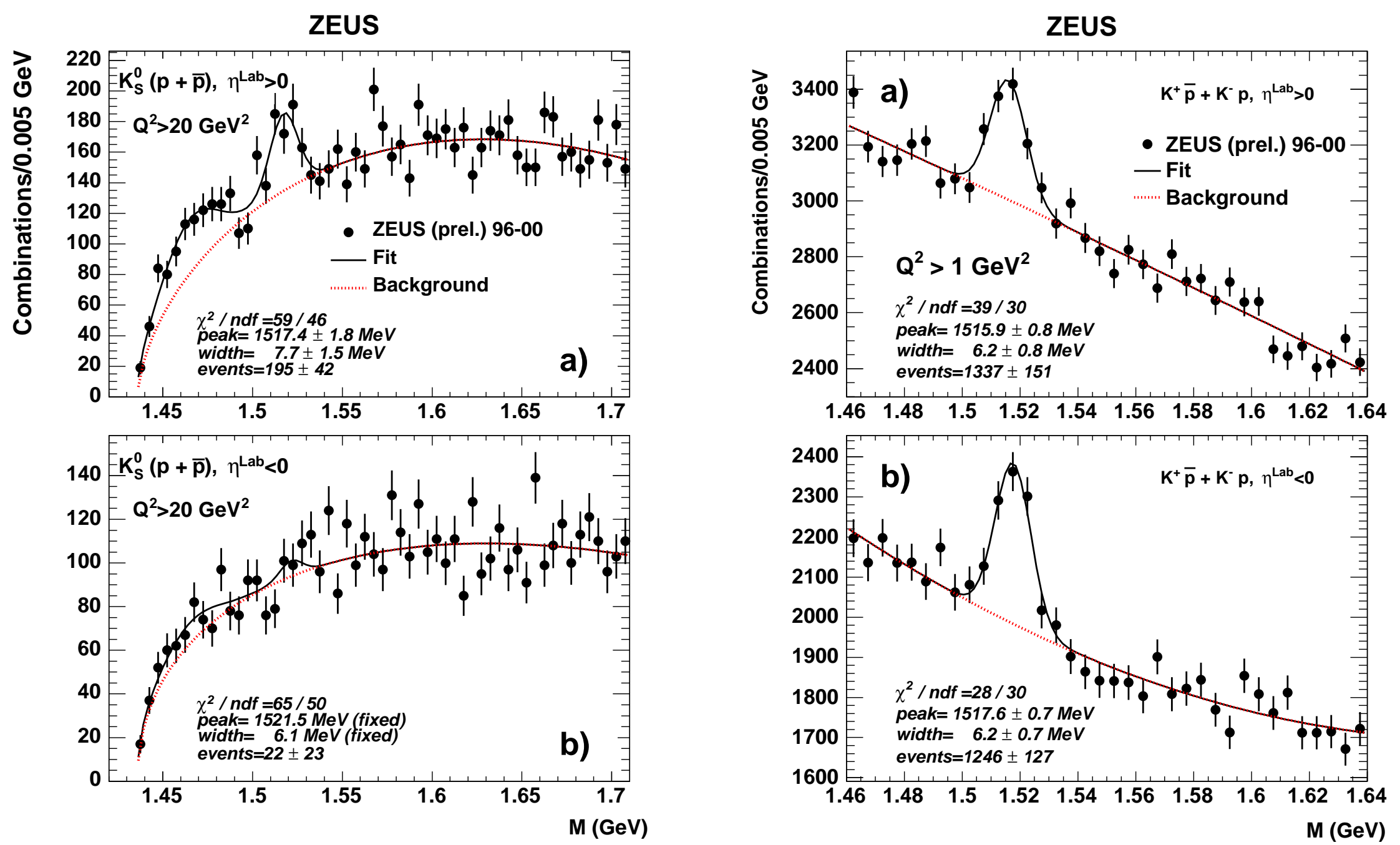

$\Theta^{ \pm}$produced mostly in forward (proton) direction

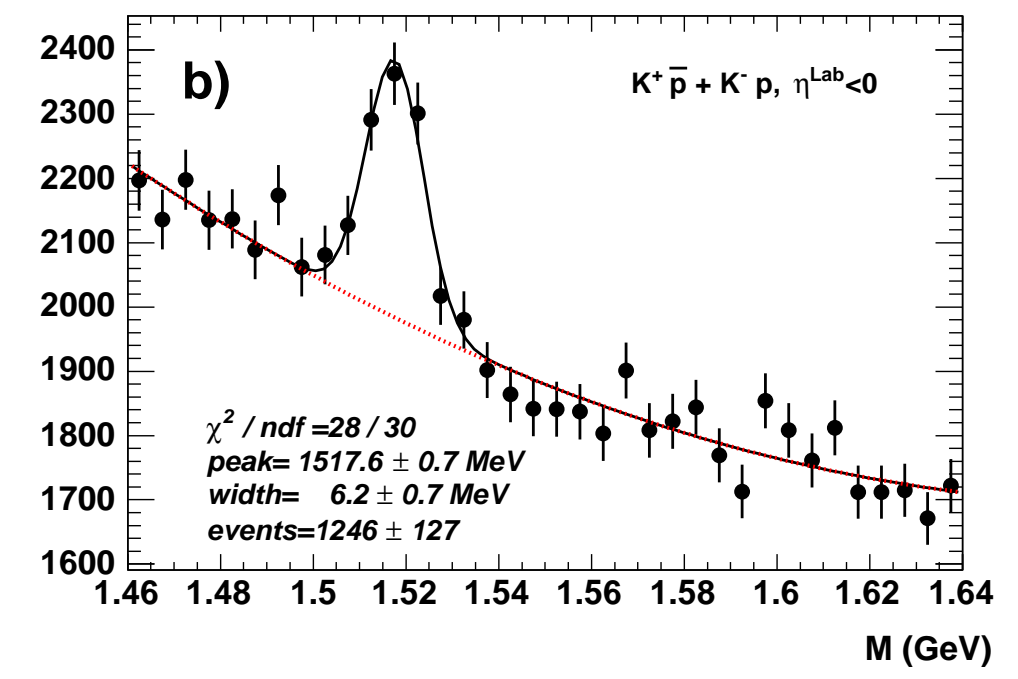

It is not a case for $\Lambda(1520)$ produced in $q / g$ fragmentation

$\Theta^{ \pm}$may have unusual production mechanism related to $p$-remnant fragmentation ? in case of coalescence, should be sizeable $d / \bar{d}$ production in DIS 


\section{$d / \bar{d}$ production in DIS with $Q^{2}>1 \mathrm{GeV}^{2}$}

\section{ZEUS}
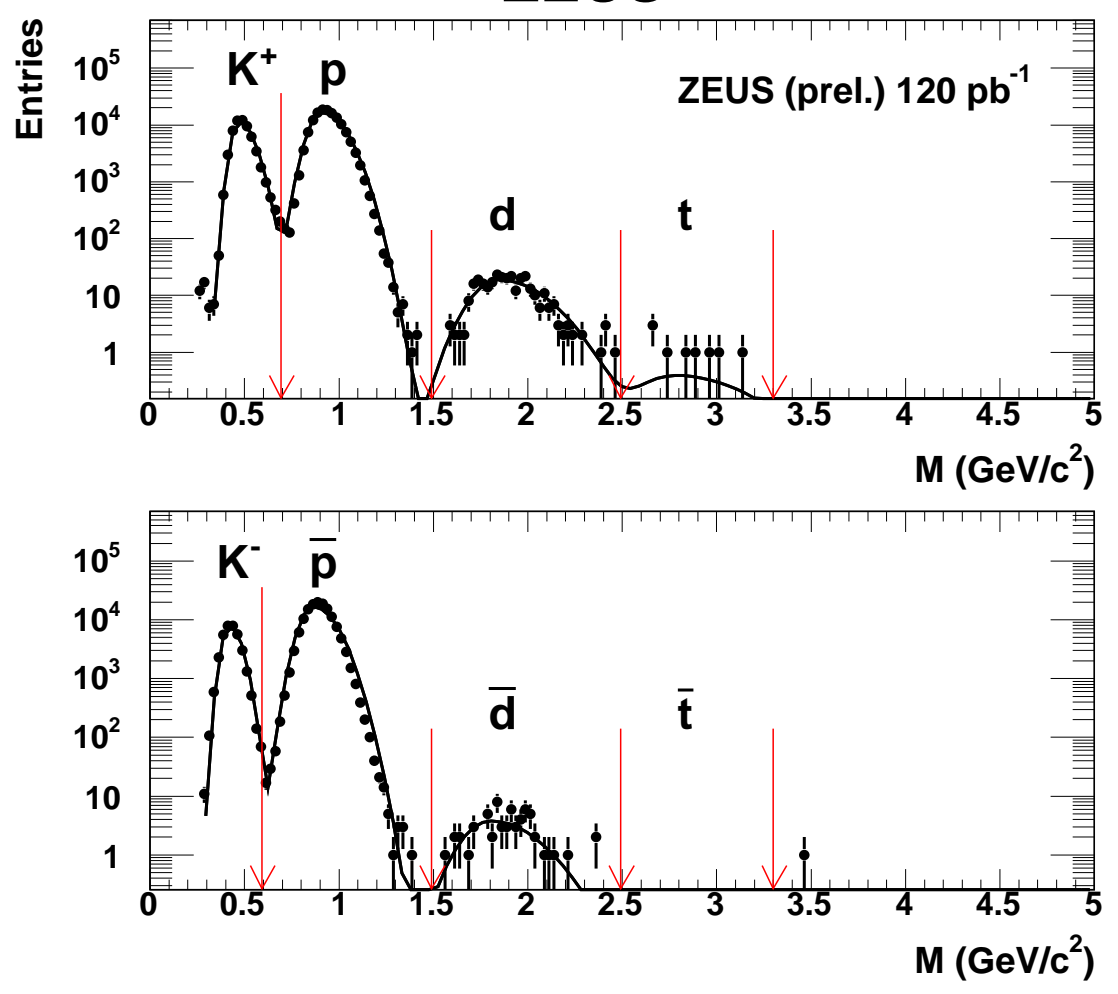

$$
M=P / f_{B B}^{-1}(d E / d x)
$$

for tracks with $d E / d x>2.5$
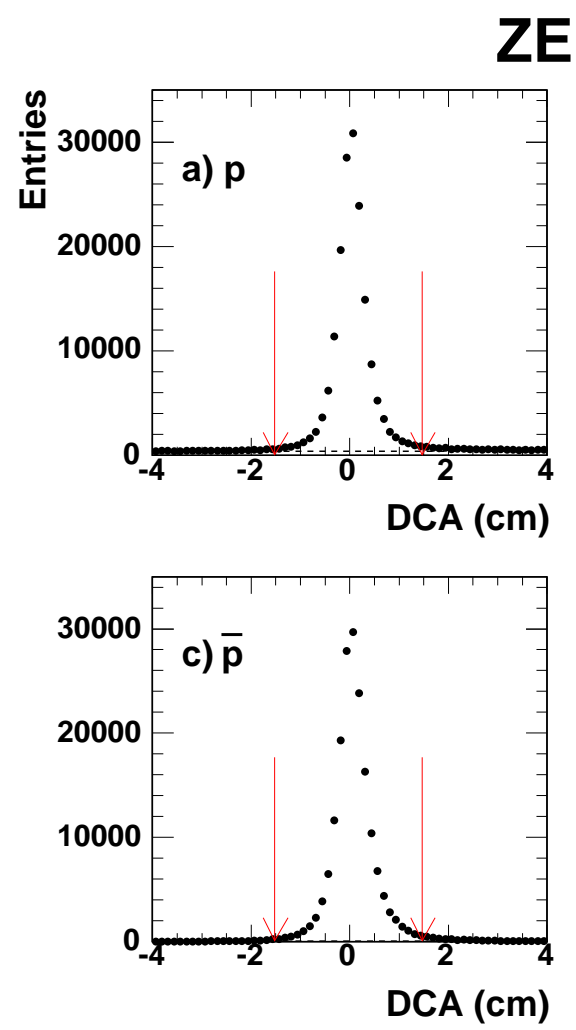
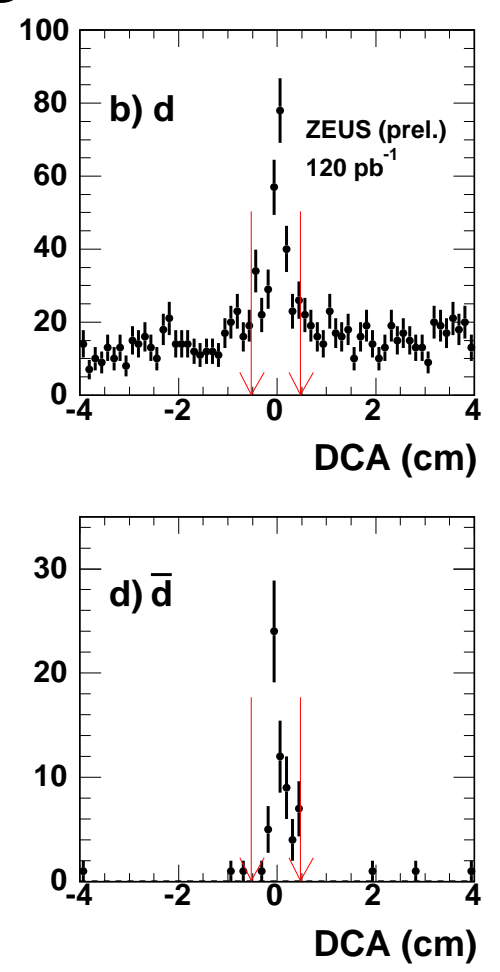

Distance of Closest Approach to beam spot in transverse plane

$$
\begin{array}{ll}
\text { Subtracting side-band background: } & N(d)=195 \pm 18 \\
& N(\bar{d})=61 \pm 8
\end{array}
$$

Yes, $d / \bar{d}$ are produced in DIS as well 


\section{$\bar{d} / \bar{p}$ ratio in DIS with $Q^{2}>1 \mathrm{GeV}^{2}$}

\section{ZEUS}

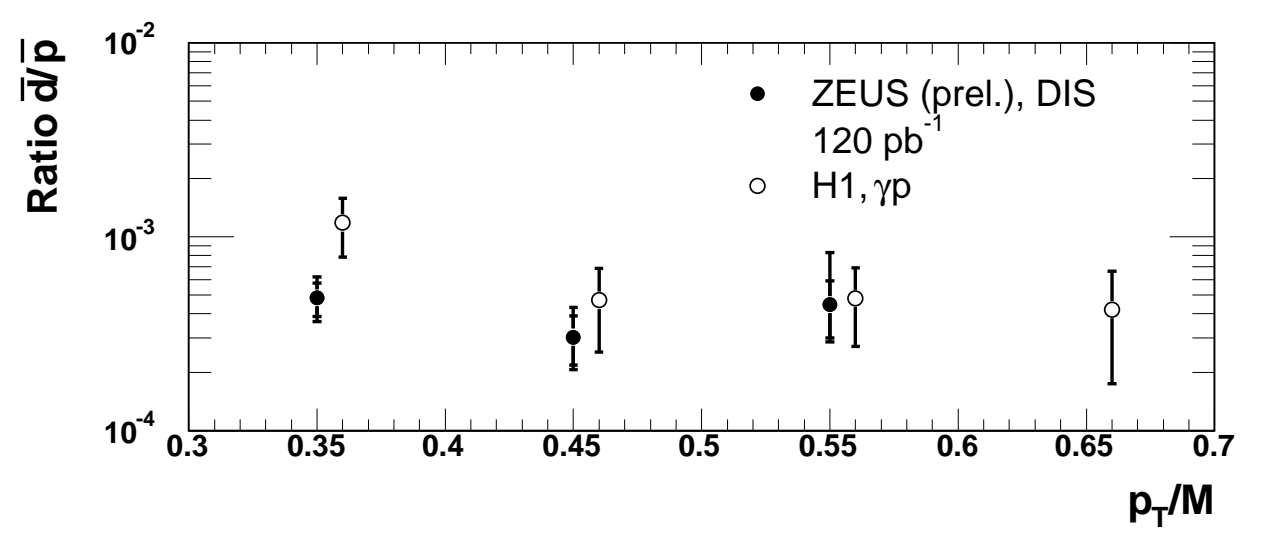

$$
\begin{array}{r}
\bar{d} / \bar{p} \text { ratio similar to } \mathrm{H} 1(\gamma p) \\
\text { and } p p \text { data }
\end{array}
$$

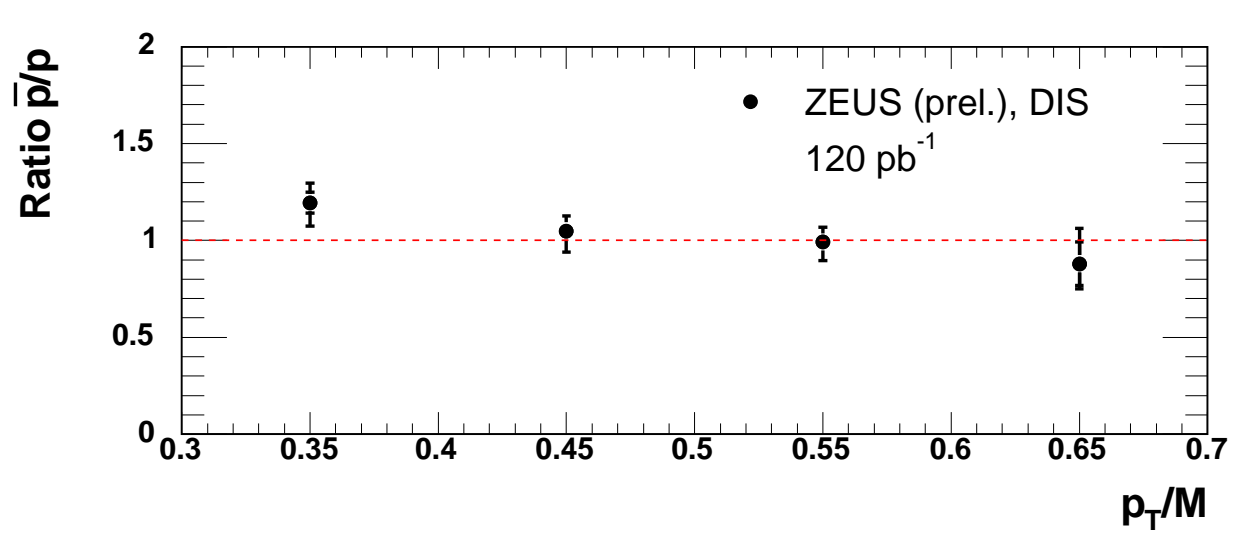

$\frac{1}{\sigma} \frac{E_{A} d^{3} \sigma_{A}}{d^{3} P_{A}}=B_{A}\left(\frac{1}{\sigma} \frac{E_{N} d^{3} \sigma_{N}}{d^{3} P_{N}}\right)^{A}$

$$
P_{N}=P_{A} / A
$$

$\bar{p} / p$ ratio $\sim 1$

Coalescence Model with $B_{2} \approx(0.5-1) \cdot 10^{-2} \mathrm{GeV}^{2}$ works for $\bar{d}$ production in DIS no $\bar{d}$ in current region of Breit frame $\Longrightarrow$ no contradiction with low $\bar{d}$ rates in $e^{+} e^{-}$

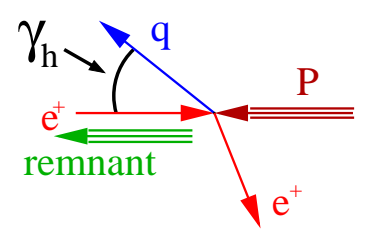

Laboratory frame

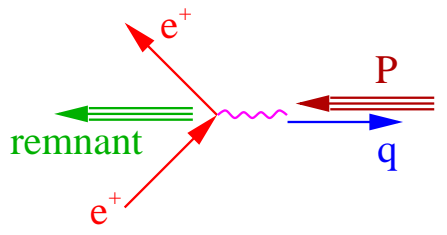

Breit frame 


\section{Charm pentaquarks}

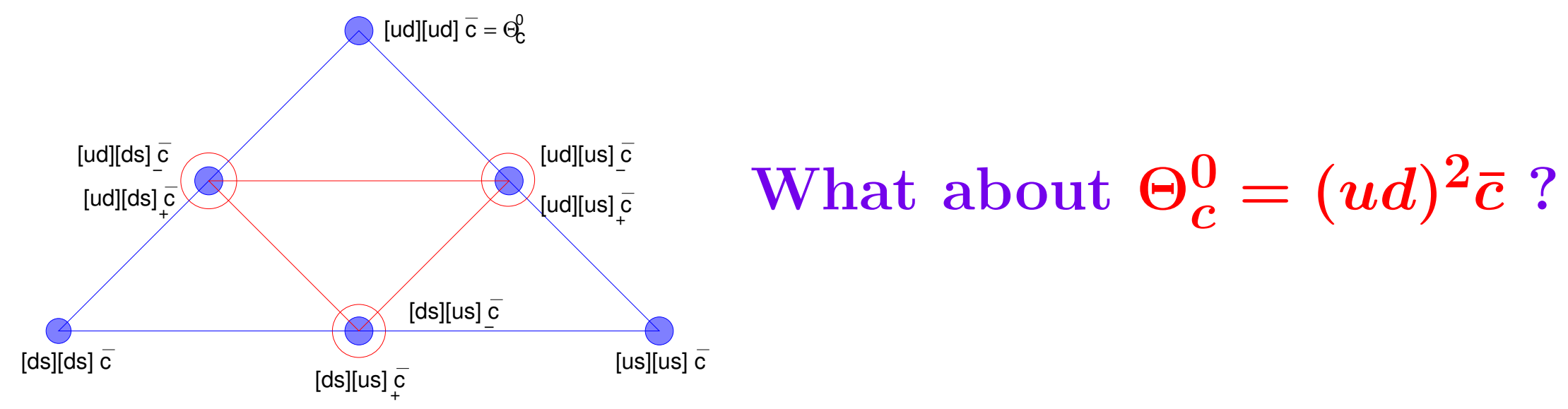

Jaffe-Wilczek (hep-ph/0307341): $M\left(\Theta_{c}^{0}\right)=2710 \mathrm{MeV}$

Such $\Theta_{c}^{0}$ would be too light to decay to $D$ mesons can decay weakly to $\Theta^{+} \pi^{-}, p K^{0} \pi^{-}, p K^{+} \pi^{-} \pi^{-}, \ldots$

Karliner-Lipkin (hep-ph/0307343): $M\left(\Theta_{c}^{0}\right)=2985 \pm 50 \mathrm{MeV}$

$$
\Gamma\left(\Theta_{c}^{0}\right) \sim 21 \mathrm{MeV}
$$

Such $\Theta_{c}^{0}$ would decay to $D^{(*)-} p(+$ c.c. $)$

H1 (hep-ex/0403017) observed a signal in $M\left(D^{*-} p\right)$ (+ c.c.)

contributed "roughly $1 \%$ to the visible $D^{* \pm}$ yield

negative results from ZEUS (hep-ex/0409033), ALEPH, BELLE, FOCUS, CDF, ... 


\section{ZEUS limits on $\Theta_{c}^{0}$ rate}

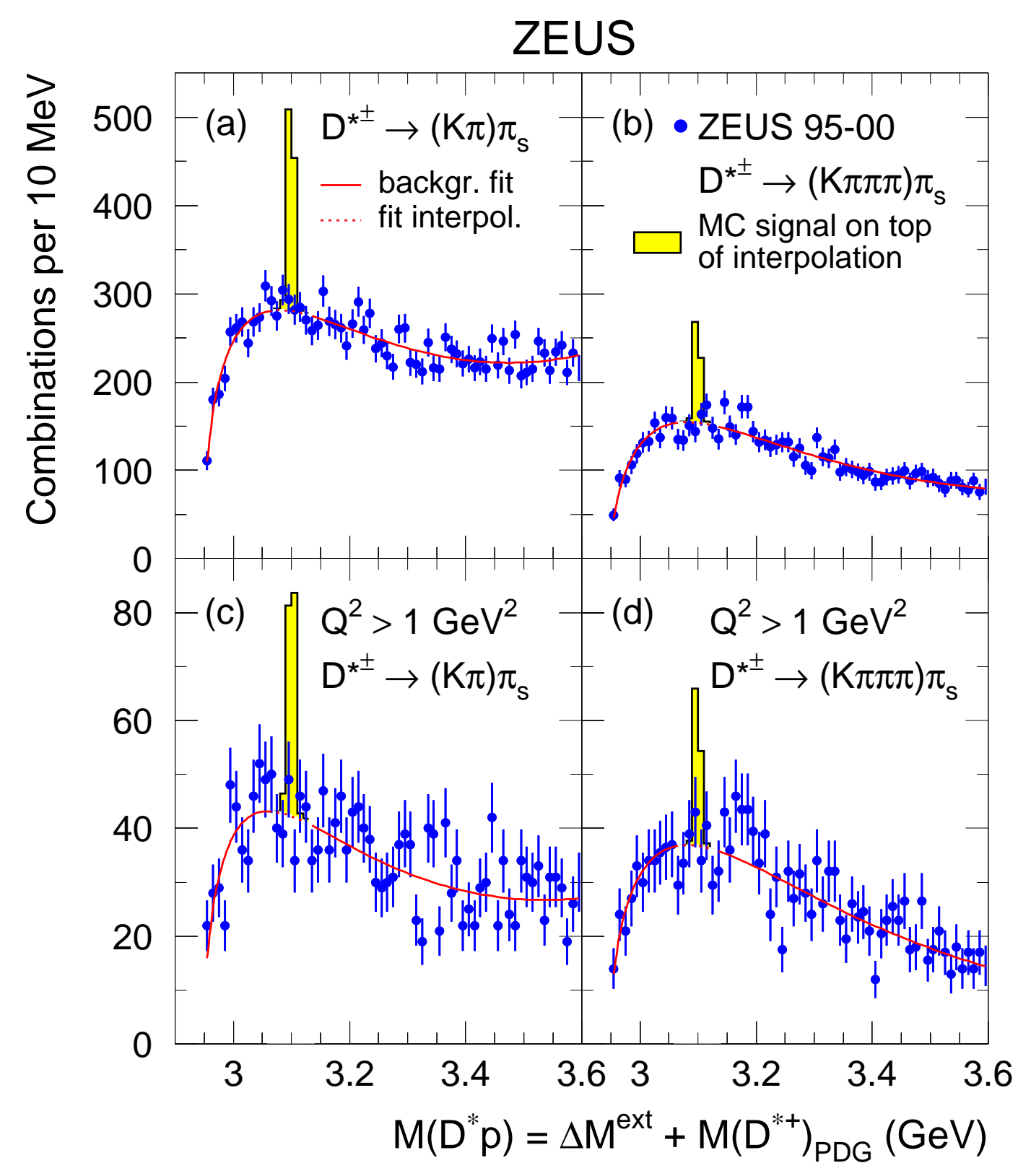

yellow signals: MC signals normalised to $1 \%$ of obs. $D^{*}$ $1 \%$ visible rate is excluded at $9 \sigma$ for full sample at $5 \sigma$ for $Q^{2}>1 \mathrm{GeV}^{2}$

95\% C.L. upper limits: visible rate $R\left(\Theta_{c}^{0} \rightarrow D^{*} p / D^{*}\right)$

$<0.23 \%$ for full sample

$<0.35 \%$ for $Q^{2}>1 \mathrm{GeV}^{2}$ acceptance corrected rate

$<0.37 \%$ for full sample $<0.51 \%$ for $Q^{2}>1 \mathrm{GeV}^{2}$ $f\left(c \rightarrow \Theta_{c}^{0}\right) \times B\left(\Theta_{c}^{0} \rightarrow D^{*} p\right)$ $<0.16 \%$ for full sample $<0.19 \%$ for $Q^{2}>1 \mathrm{GeV}^{2}$ 


\section{H1 and ZEUS results on $\Theta_{c}^{0} \rightarrow D^{*} p$ disagree}
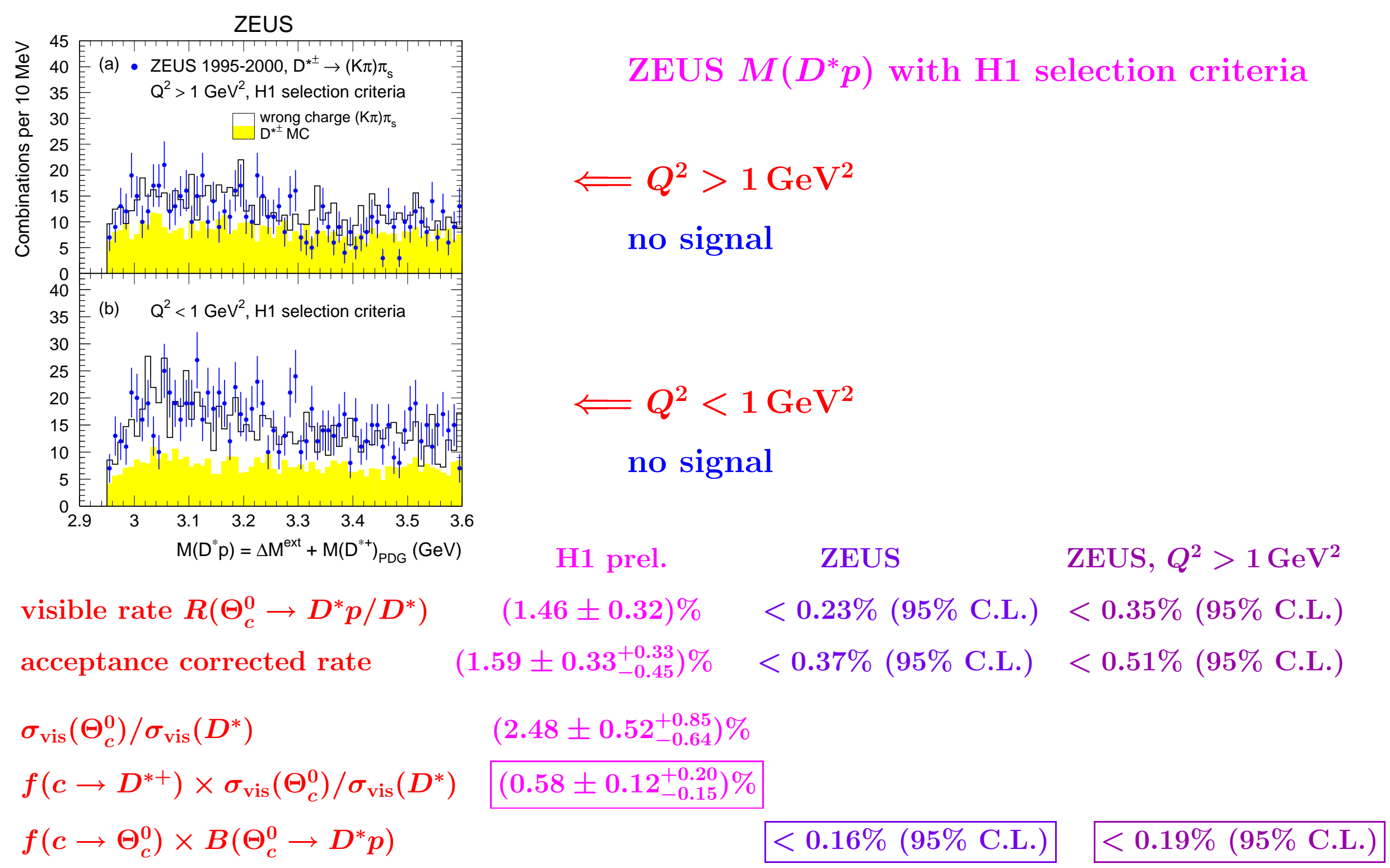

\section{HERA II data can help to resolve the disagreement}




\section{Study of excited $D$ mesons at HERA}

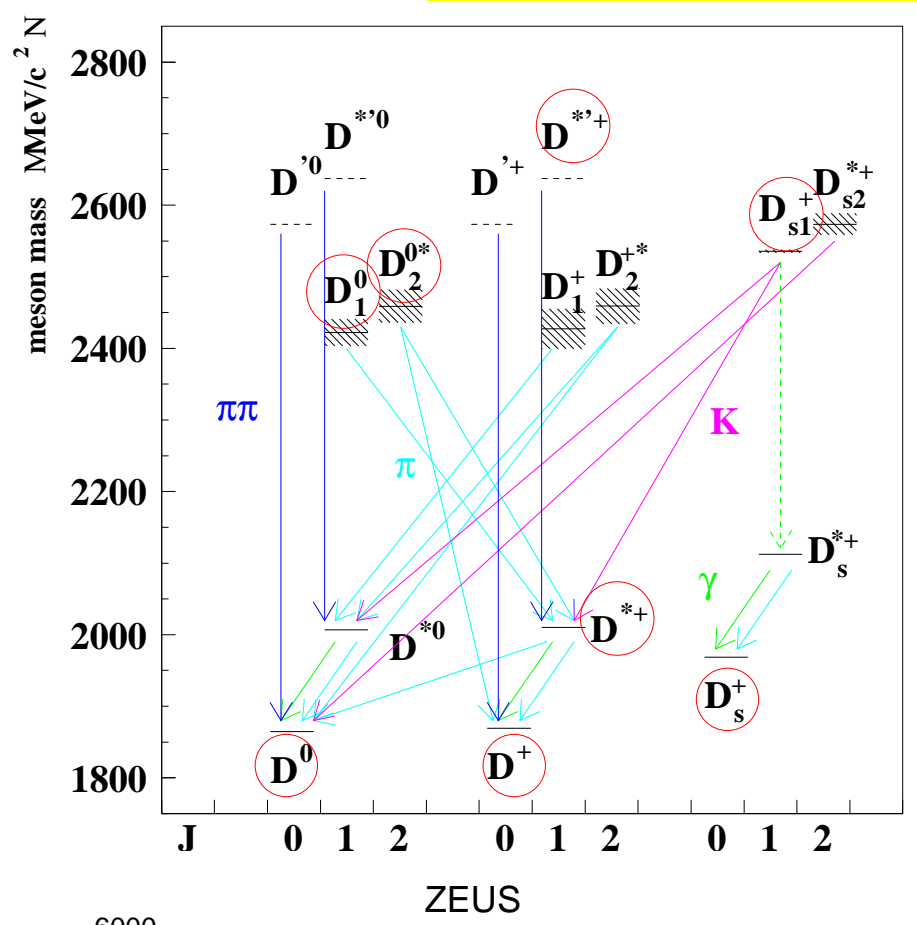

Orbitally excited:

1) $D_{1}^{0}, D_{2}^{* 0} \rightarrow D^{*+} \pi^{-}$(+ c.c. $)$

2) $D_{s 1}^{+} \longrightarrow D^{*+} K^{0}$ (+ c.c. $) \Longrightarrow$ discussion

Search for radially excited:

3) $D^{* \prime+} \rightarrow D^{*+} \pi^{+} \pi^{-}$(+ c.c.)

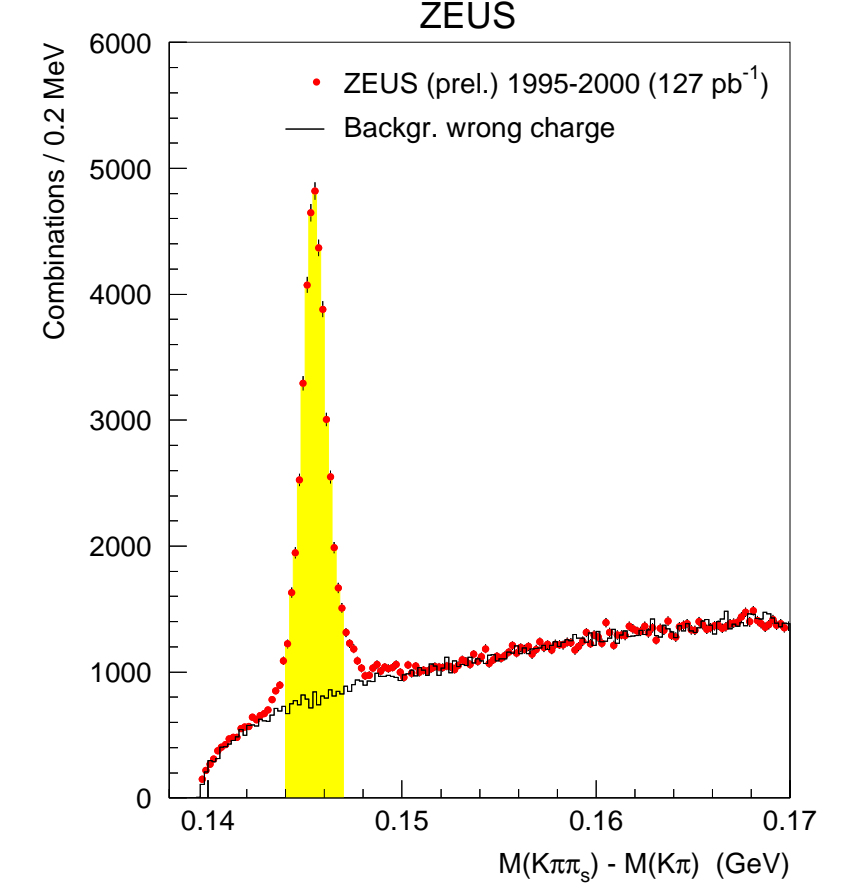

$D^{*+} \rightarrow D^{0} \pi_{s}^{+} \rightarrow\left(K^{-} \boldsymbol{\pi}^{+}\right) \boldsymbol{\pi}_{s}^{+}$(+ c.c.)

$\Delta M=M\left(D^{*+}\right)-M\left(D^{0}\right) \sim m_{\pi}$

$P_{\perp}^{D^{*}}>2 \mathrm{GeV}$ and $-1.5<\eta^{D^{*}}<1.5$

In the yellow band under background:

$$
N\left(D^{* \pm}\right)=31350 \pm 240
$$




\section{Charm-strange $D_{s 1}^{ \pm}(2536)$ meson}

$$
\underline{D_{s 1}^{ \pm}(2536) \rightarrow D^{* \pm} K_{s}^{0}, \quad K_{s}^{0} \rightarrow \pi^{+} \pi^{-}}
$$

$\Delta M^{e x t}=M\left(K \pi \pi_{S} \pi_{3} \pi_{4}\right)-M\left(K \pi \pi_{s}\right)-M\left(\pi_{3} \pi_{4}\right)$

$$
N\left(D_{s 1}^{+}\right)=62.3 \pm 9.3
$$$$
M\left(D_{s 1}^{+}\right)=2534.2 \pm 0.6 \pm 0.5 \mathrm{MeV} \quad\left(\sim M_{\mathrm{PDG}}\right)
$$

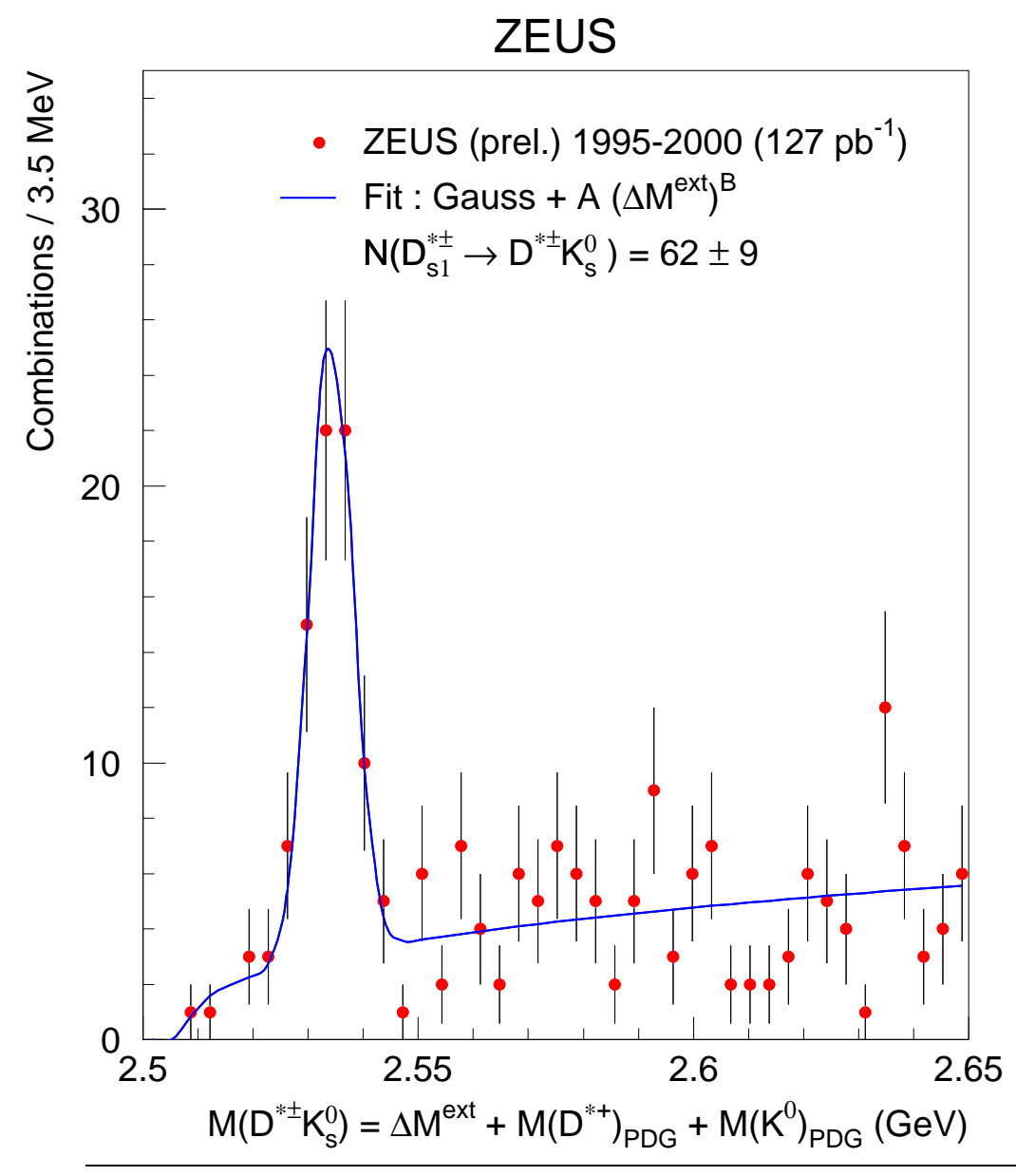

"Charm spectroscopy and exotics at ZEUS"

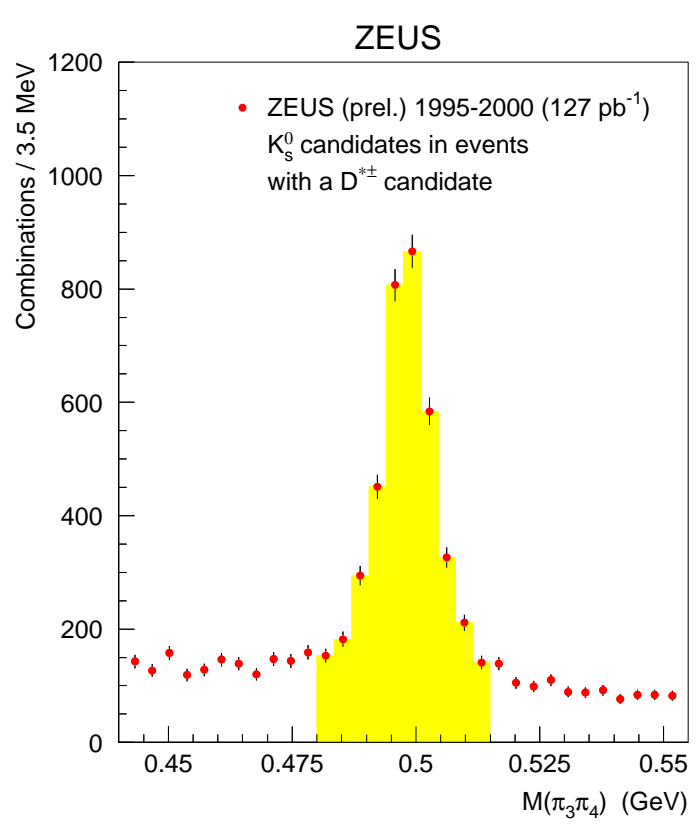

Helicity angle $\alpha$ : between $K_{s}^{0}$ and $\pi_{s}$ in $D^{* \pm}$ r.f. Fit to a form : $1+R \cos ^{2} \alpha$ $R=-0.53 \pm 0.32$ (stat.) ${ }_{-0.14}^{+0.05}$ (syst.) (ZEUS prel.)

CLEO $\left(D_{s 1}^{+} \rightarrow D^{* 0} K^{+}\right): R=-0.23_{-0.32}^{+0.40}$

ZEUS : consistent with $R=0$, i.e. $J^{P}=1^{+}$ does not contradict to $R=-1$ expected for $1^{-}, 2^{+}$ Belle (recent) : $R=-0.70 \pm 0.03 \rightarrow$ mixture of $D$ and $S$ waves due to interf. with $D_{s J}^{+}(2460)$ ? 


\section{Fragmentation fractions for excited $D$ mesons}

Using world average for $f\left(c \rightarrow D^{*+}\right)$ :

\begin{tabular}{|c|c|c|c|}
\hline & $f\left(c \rightarrow D_{1}^{0}\right)[\%]$ & $f\left(c \rightarrow D_{2}^{* 0}\right)[\%]$ & $f\left(c \rightarrow D_{s 1}^{+}\right)[\%]$ \\
\hline \hline ZEUS (prel.) $)$ & $1.46 \pm 0.18_{-0.27}^{+0.33} \pm 0.06$ & $2.00 \pm 0.58_{-0.48}^{+1.40} \pm 0.41$ & $1.24 \pm 0.18_{-0.06}^{+0.08} \pm 0.14$ \\
\hline CLEO & $1.8 \pm 0.3$ & $1.9 \pm 0.3$ & \\
\hline OPAL & $2.1 \pm 0.8$ & $5.2 \pm 2.6$ & $1.6 \pm 0.4 \pm 0.3$ \\
\hline ALEPH & $1.6 \pm 0.5$ & $4.7 \pm 1.0$ & $0.94 \pm 0.22 \pm 0.07$ \\
\hline DELPHI & $1.9 \pm 0.4$ & $4.7 \pm 1.3$ & \\
\hline
\end{tabular}

1) the same amounts of excited $D$ mesons in $e^{+} e^{-}$and $e p$ data

2) situation with $f\left(c \rightarrow D_{2}^{* 0}\right)$ is not clear

3) $f\left(c \rightarrow D_{s 1}^{+}\right)$is twice as large as the expectation :

$$
\gamma_{s} \times f\left(c \rightarrow D_{1}^{0}\right) \approx 0.3 \times 2 \%=0.6 \%
$$

Why $f\left(c \rightarrow D_{s 1}^{+}\right)$is so large ?

Is it connected with its strange helicity ? 


\section{Summary and Outlook}

- $\Theta^{+} \rightarrow K_{s}^{0} p$ production observed in high- $Q^{2}$ DIS by ZEUS.

H1 does not see the signal that is not in statistical contradiction with ZEUS. Studies suggest $\Theta^{+}$production in ep related to proton-remnant fragmentation

- Sizeable $d / \bar{d}$ production observed in DIS.

$\bar{d} / \bar{p}$ ratio in DIS similar to $\gamma p(\mathrm{H} 1)$ and $p p$ data, corresponds to Coalescence Model with $B_{2} \approx(0.5-1) \cdot 10^{-2} \mathrm{GeV}^{2}$

- H1 and ZEUS results on $\Theta_{c}^{0} \rightarrow D^{*} p$ disagree.

Using larger statistics, ZEUS does not see a signal observed by H1

- Rates of excited $D^{* *}$ mesons are close in $e^{+} e^{-}$and $e p$ data. $D_{s 1}^{ \pm}(2536)$ shows questionable helicity distribution and "too large" $f\left(c \rightarrow D_{s 1}^{+}\right)$

\section{Expect more from HERA II}

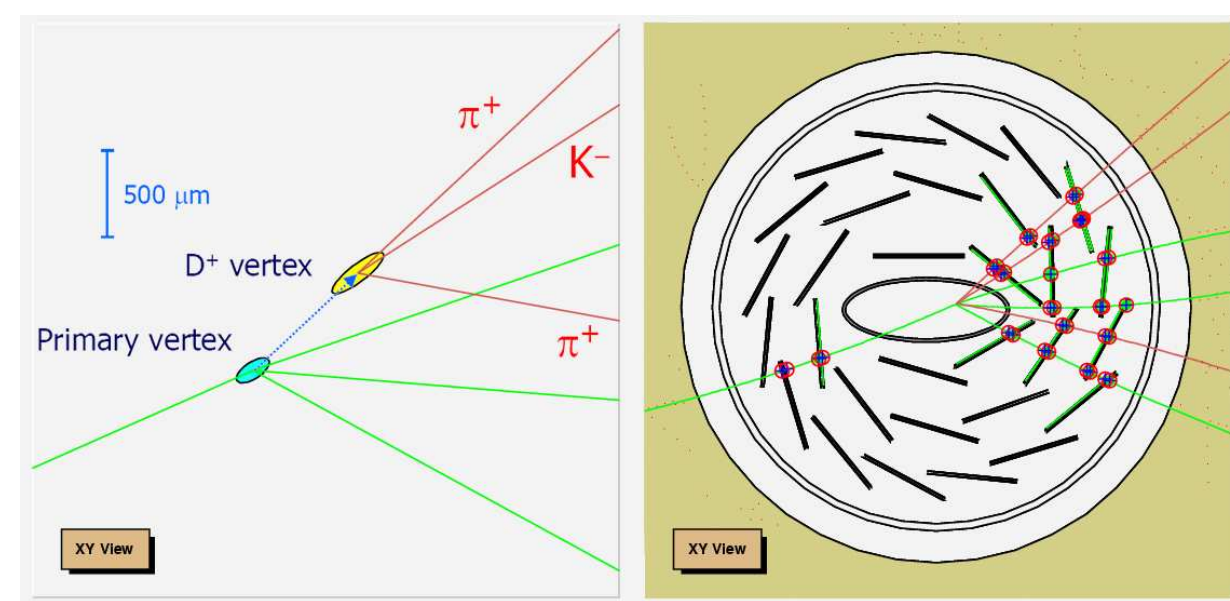

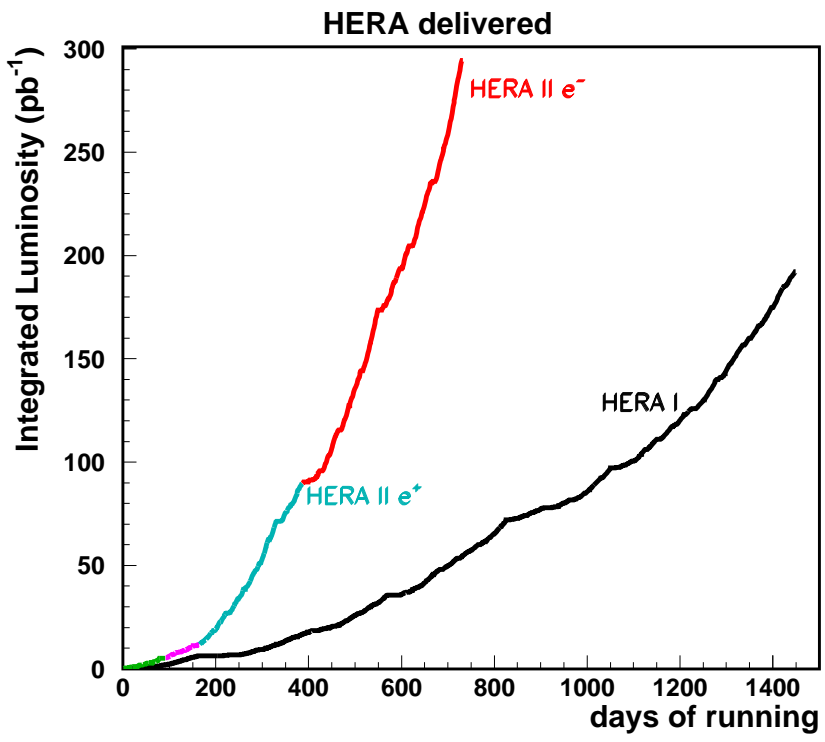




\section{$M\left(K_{s}^{0} p(\bar{p})\right)$}

\section{ZEUS}
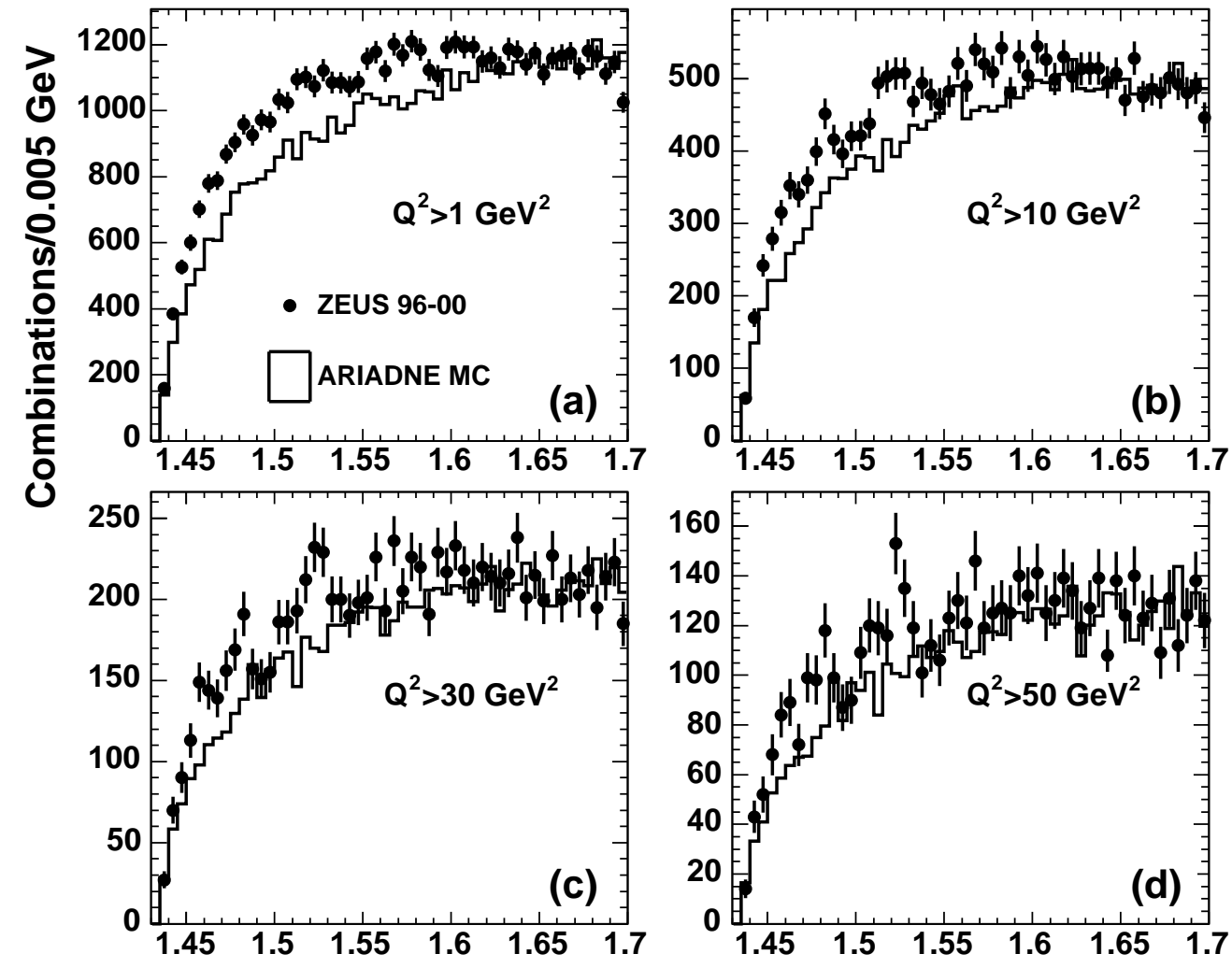

large background

signal becomes visible

for $Q^{2}>10 \mathrm{GeV}^{2}$

ARIADNE (JETSET) MC

(normalized to data above $1.65 \mathrm{GeV}$ ) does not reproduce the shape.

$$
\Sigma(1480), \Sigma(1560) \text { bumps ? }
$$
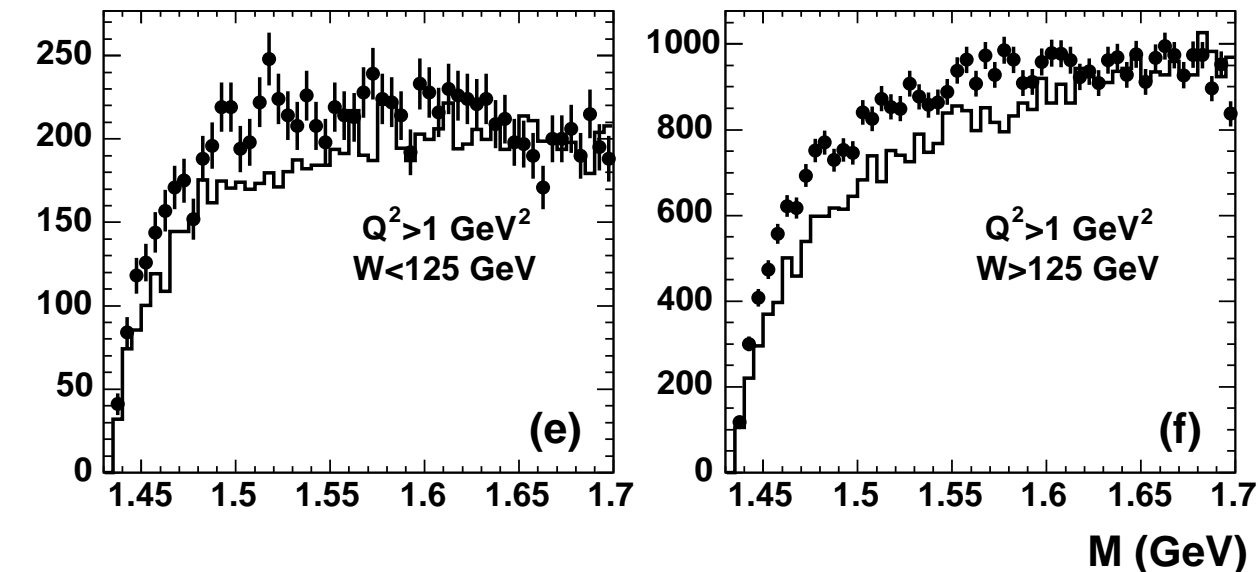

for $Q^{2}>1 \mathrm{GeV}^{2}$,

signal is visible

for $W<125 \mathrm{GeV}$ 


\section{$\Lambda_{c}$ and $\Lambda(1520)$}

\section{ZEUS}
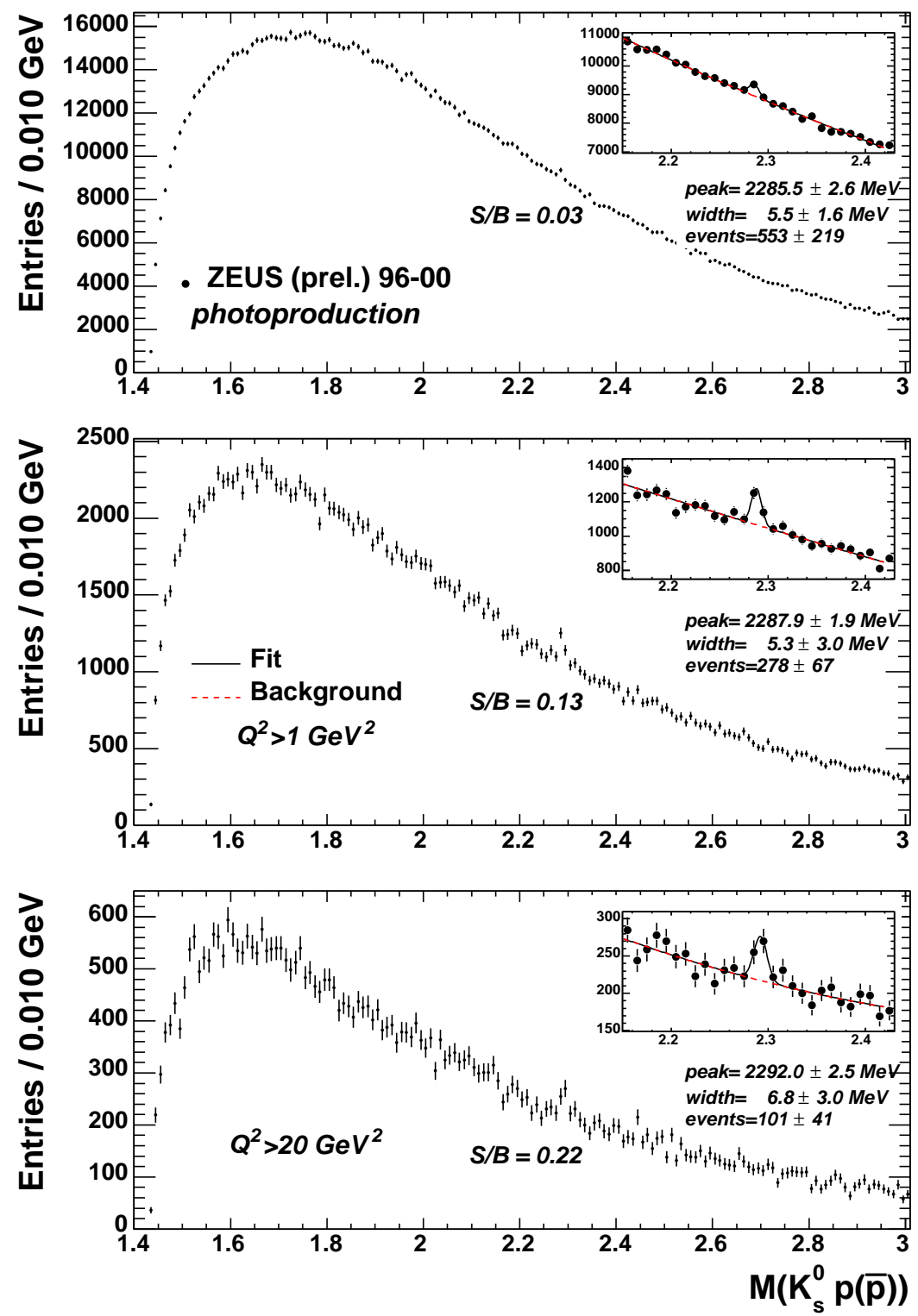

$\Lambda_{c}$ seen better for $Q^{2}>20 \mathrm{GeV}^{2}$

\section{ZEUS}
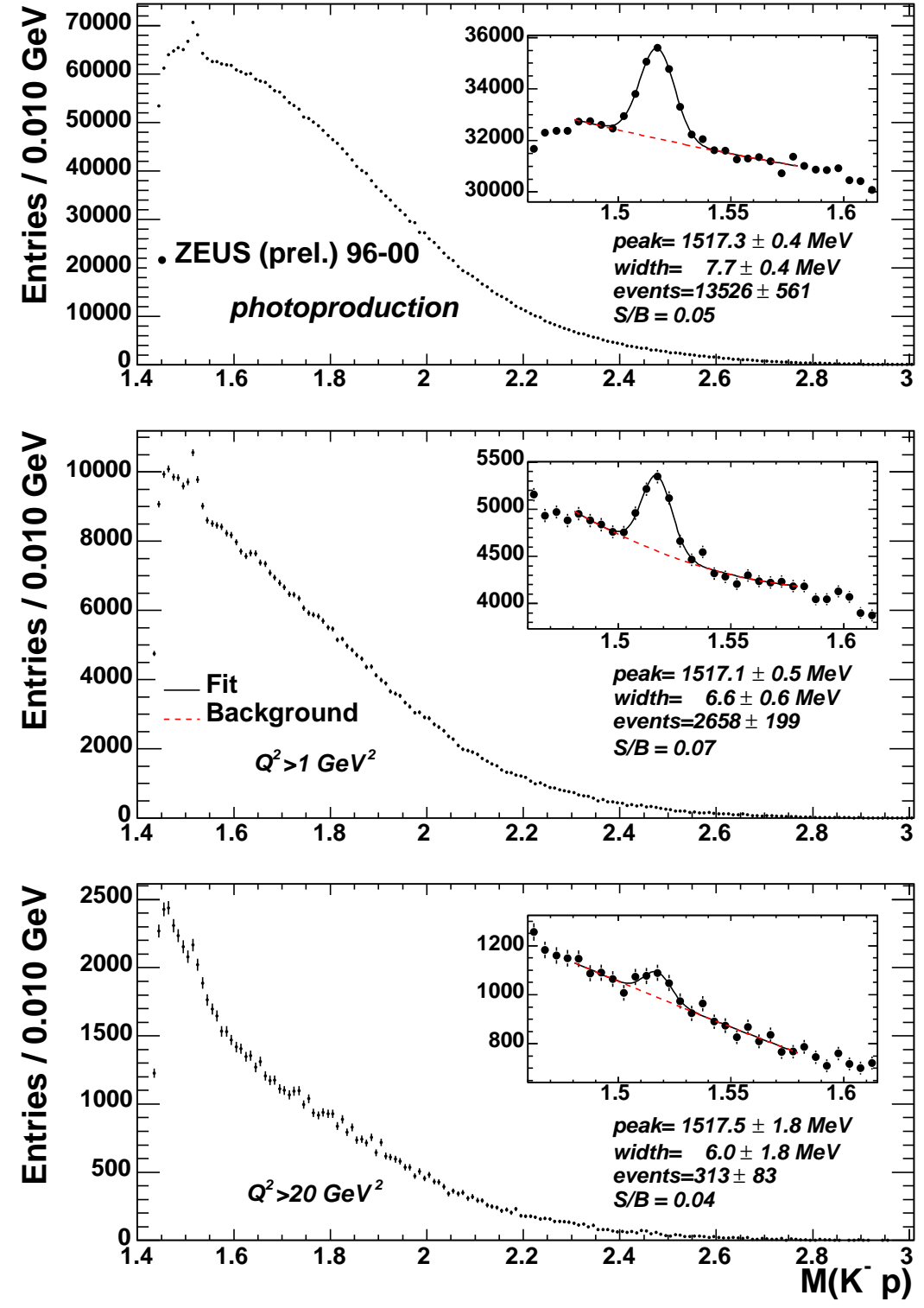

It is not a case for $\Lambda(1520)$ 


\section{Search for $\Theta^{++} \rightarrow K^{+} p(+$ c.c. $)$}

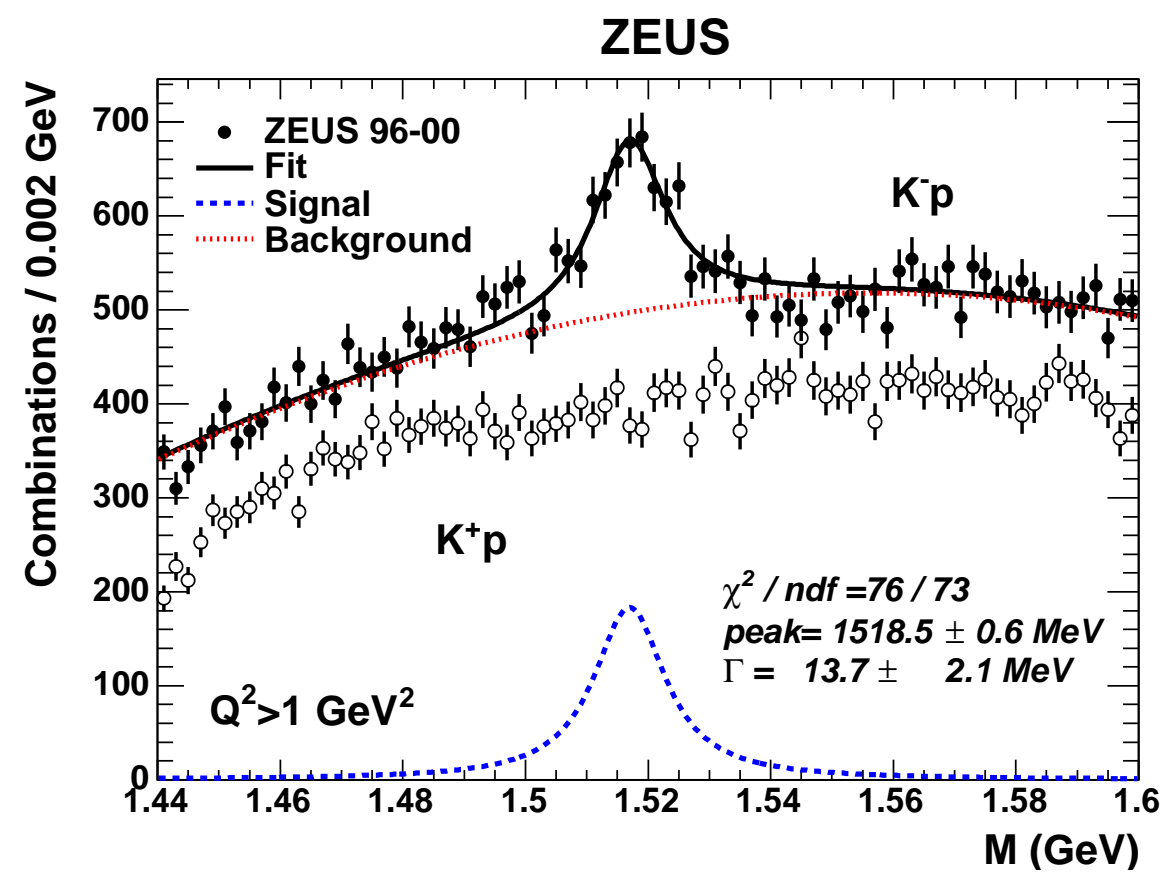

For $N K$ bound state,

both $I=0,1$ are possible

$I=1$ : triplet $\Theta^{0}, \Theta^{+}, \Theta^{++}$

$\Longleftarrow$ search for $\Theta^{++} \rightarrow K^{+} p(+$ c.c. $)$ no signal

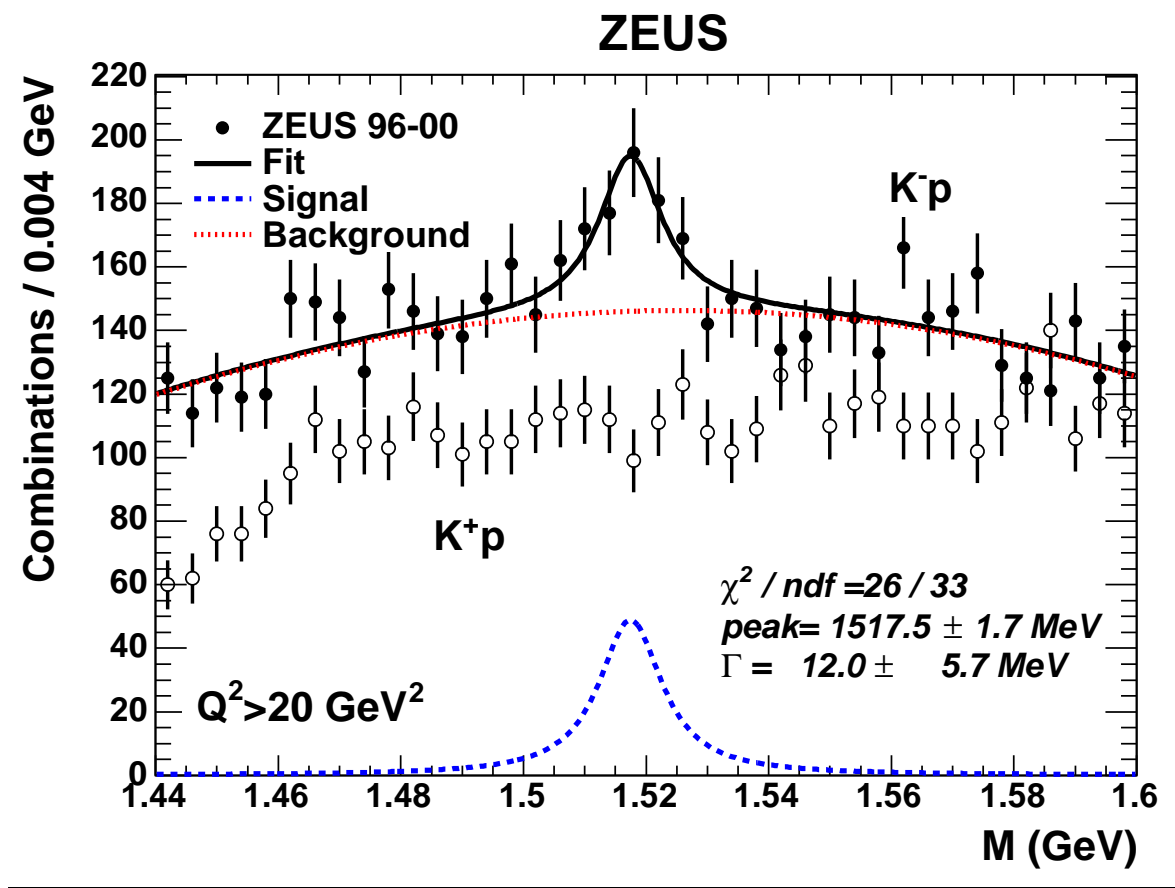

$\Longleftarrow$ no $\Theta^{++}$signal for $Q^{2}>20 \mathrm{GeV}^{2}$ as well

Does not contradict to $\Theta^{++}$observation by STAR with $R\left(\Theta^{++} / \Lambda(1520)\right) \approx 0.1 \%$ 


\section{Search for pentaquarks with $S= \pm 2$}

\section{ZEUS}

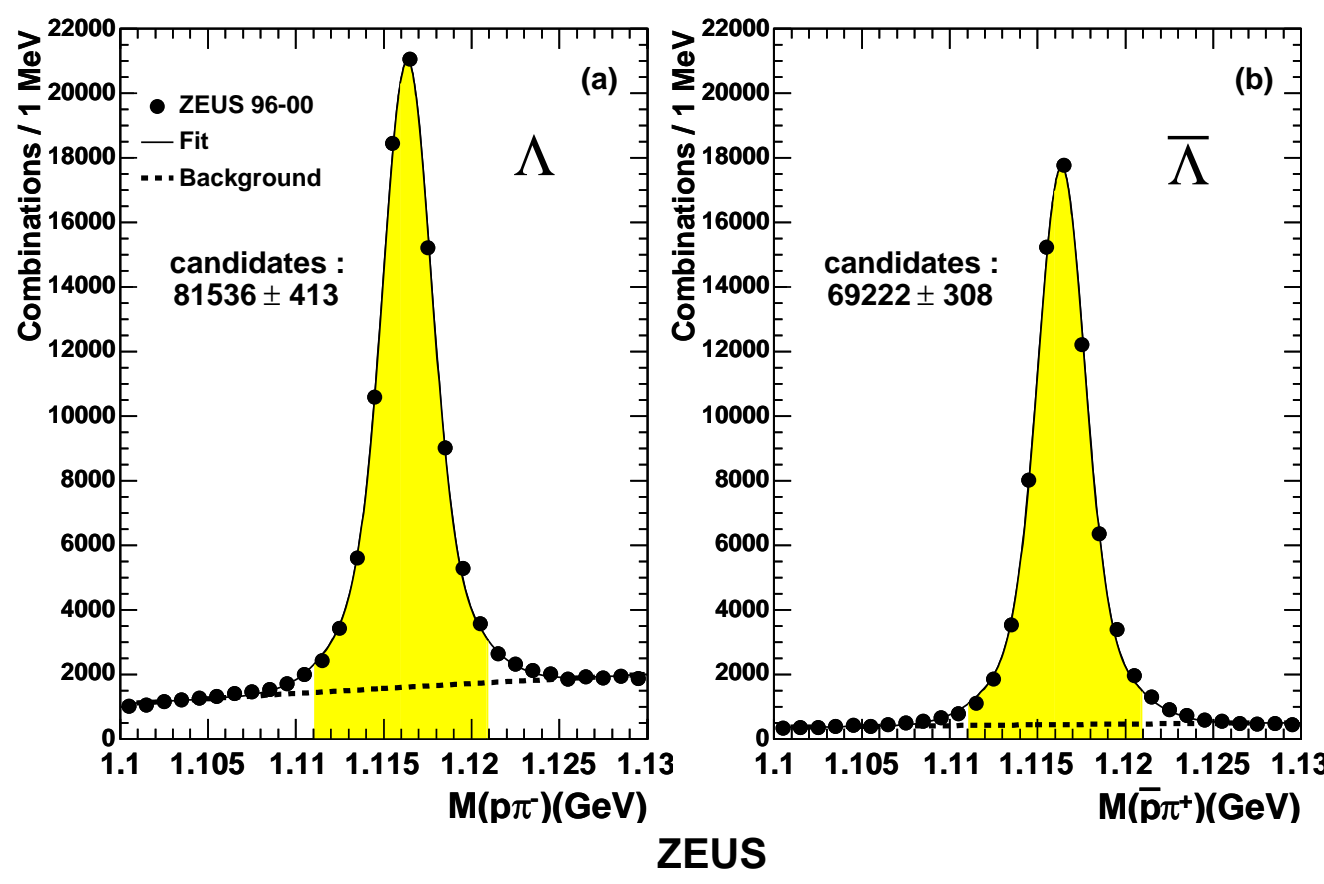

$\Xi_{3 / 2}^{--, 0} \rightarrow \Xi \pi$ observed by NA49

ZEUS search in DIS, $Q^{2}>1 \mathrm{GeV}^{2}$

$\Lambda^{0} \rightarrow p \pi^{-}$(+c.c.) are well identified using the displaced vertices

$\Longleftarrow \sim 150000$ candidates
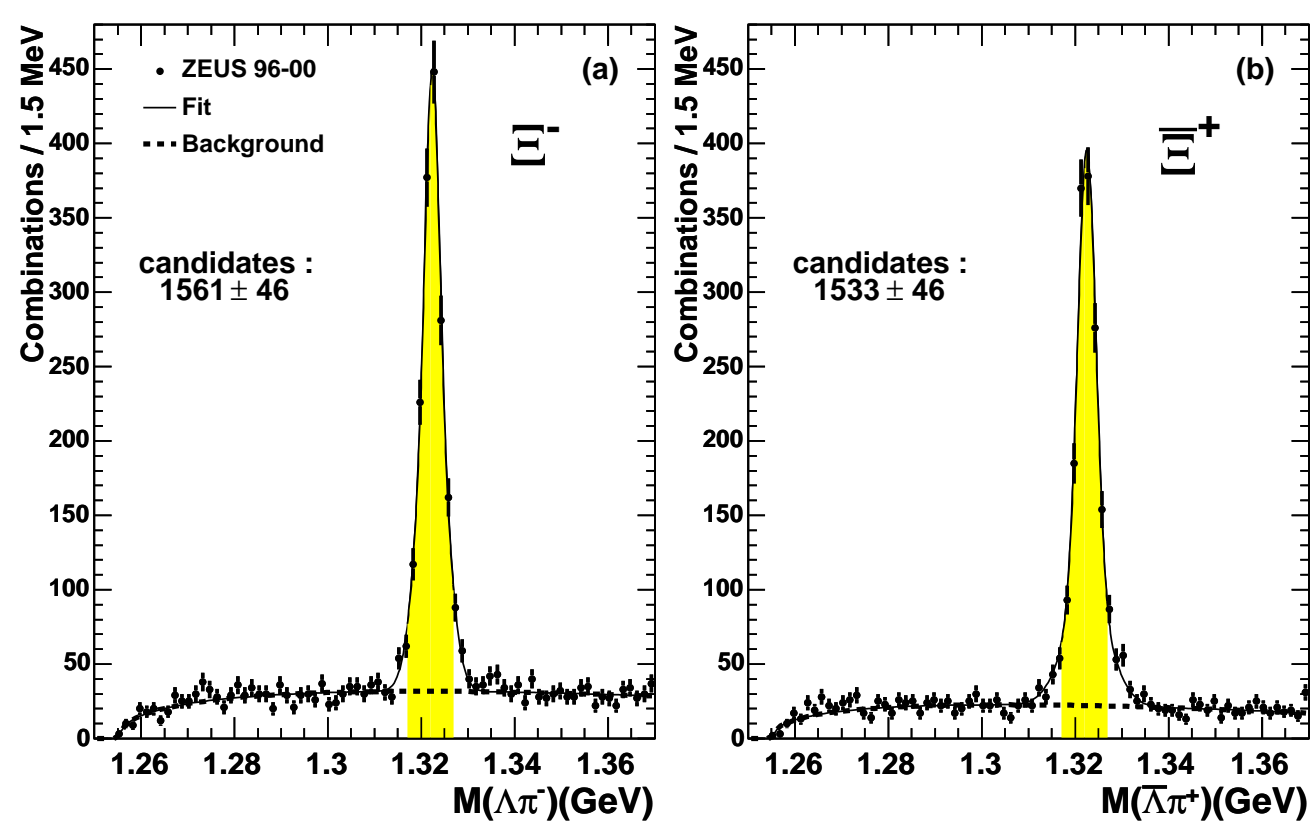

Combining with additional track

$\Xi^{-} \rightarrow \Lambda^{0} \pi^{-}$(+c.c.)

$\Longleftarrow \sim 3000$ candidates 


\section{$M(\Xi \pi)$ and upper limit on $R\left(\Xi_{3 / 2}^{--, 0} / \Xi^{0}(1530)\right)$}

\section{ZEUS}

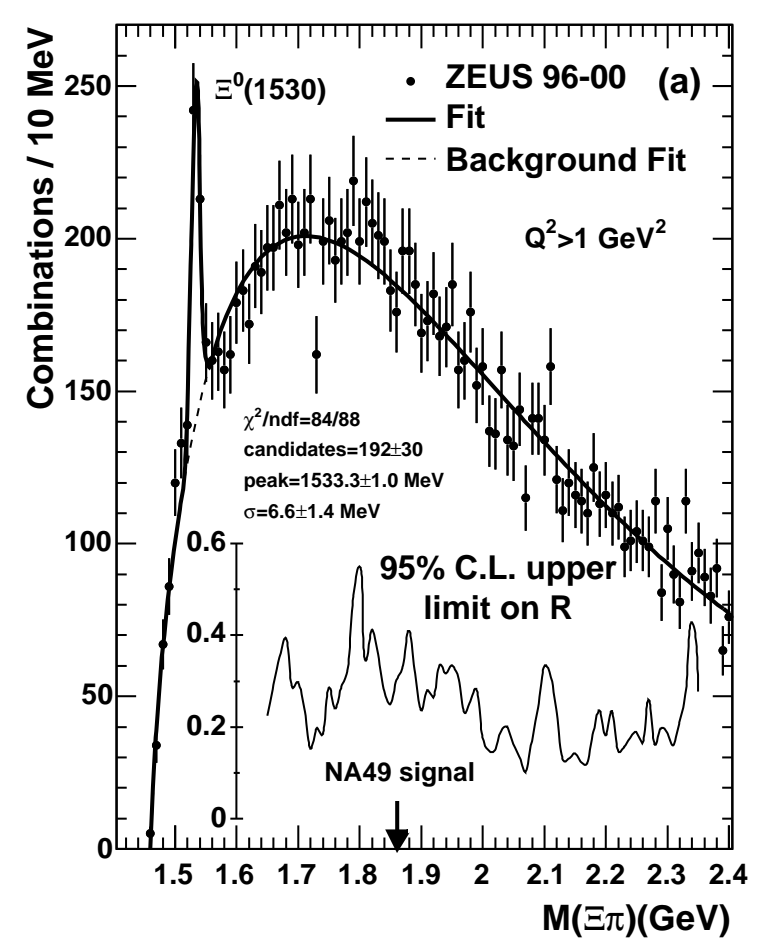

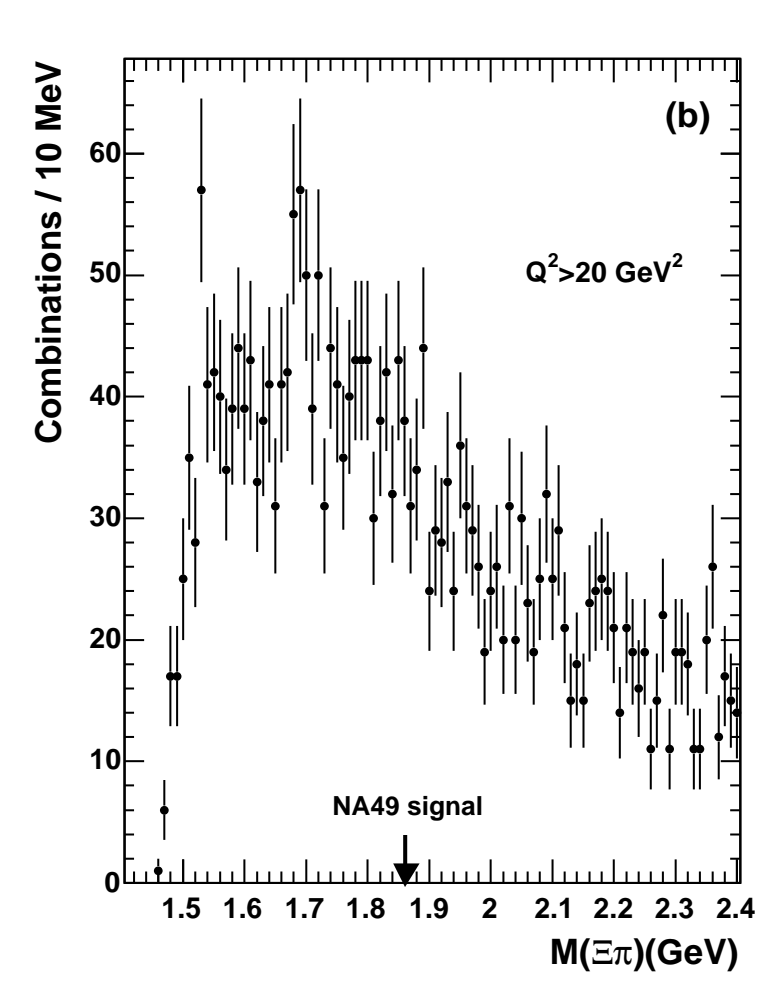

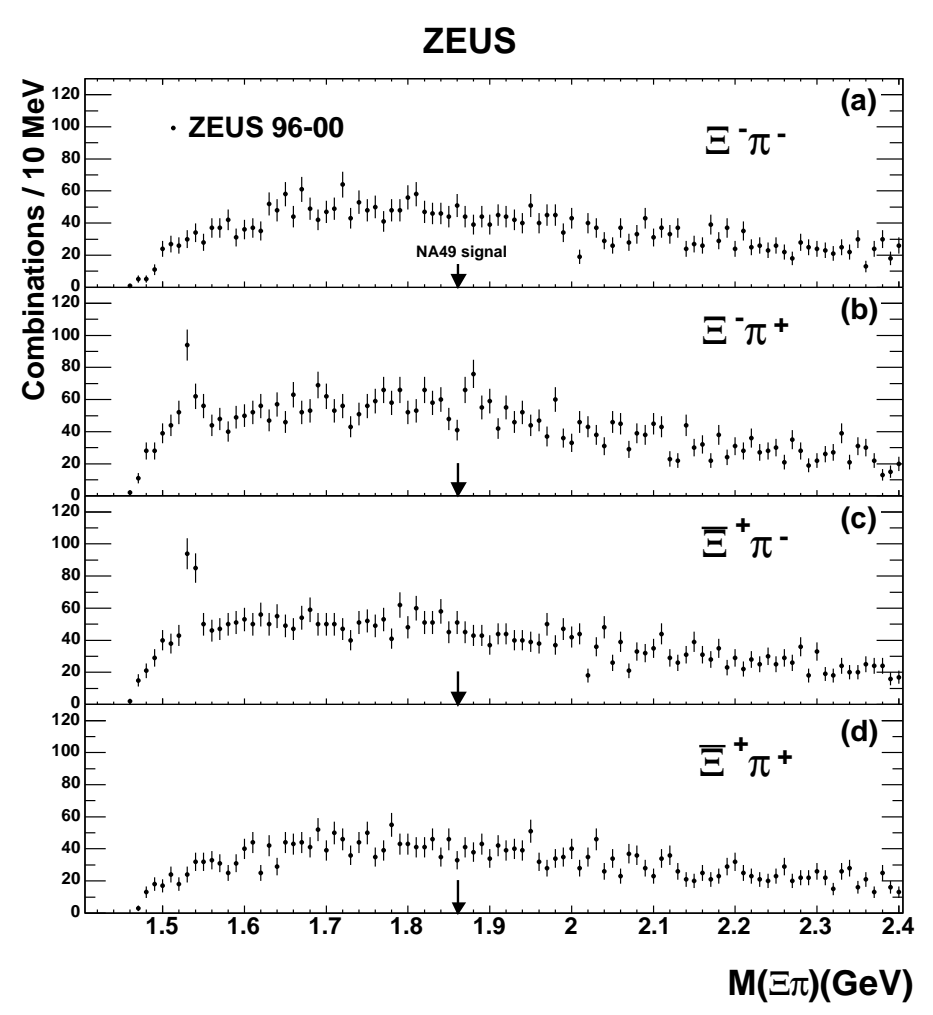

approx. the same number of $\Xi^{0}(1530) \rightarrow \Xi^{-} \pi^{+}$as in NA49

No $\Xi_{3 / 2}$ signal for $Q^{2}>1 \mathrm{GeV}^{2}$ and $Q^{2}>20 \mathrm{GeV}^{2}$; in all charge combinations $\boldsymbol{R}\left(\Xi_{3 / 2}^{--, 0} / \Xi^{0}(1530)\right)<0.29(95 \%$ C.L. $)$ in the NA49 signal region

Note: ZEUS studies central production

NA49 covers forward production 


\section{$D^{* \pm}$ reconstruction for charm pentaquark searches}

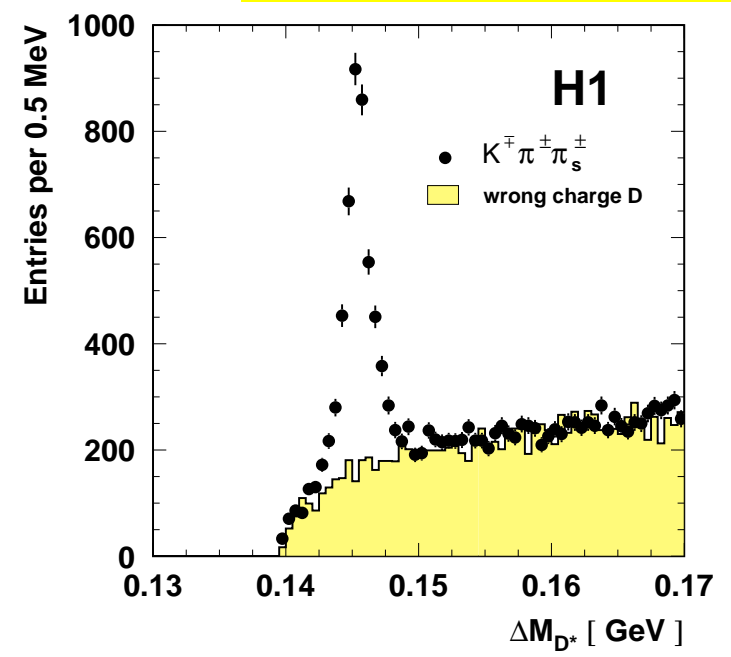

\section{H1 96-00 data $\left(75 \mathrm{pb}^{-1}\right)$}

$$
\begin{aligned}
& Q^{2}>1 \mathrm{GeV}^{2}, 0.05<y<0.7 \\
& P_{T}\left(D^{* \pm}\right)>1.5 \mathrm{GeV},-1.5<\eta\left(D^{* \pm}\right)<1.0 \\
& N\left(D^{* \pm}\right) \sim 3400 \\
& \left(\text { for } Q^{2}<1 \mathrm{GeV}^{2}: N\left(D^{* \pm}\right) \sim 4900\right) \\
& \quad \text { ZEUS 95-00 data }\left(126 \mathrm{pb}^{-1}\right)
\end{aligned}
$$

\section{ZEUS 95-00 data $\left(126 \mathrm{pb}^{-1}\right)$}

ZEUS

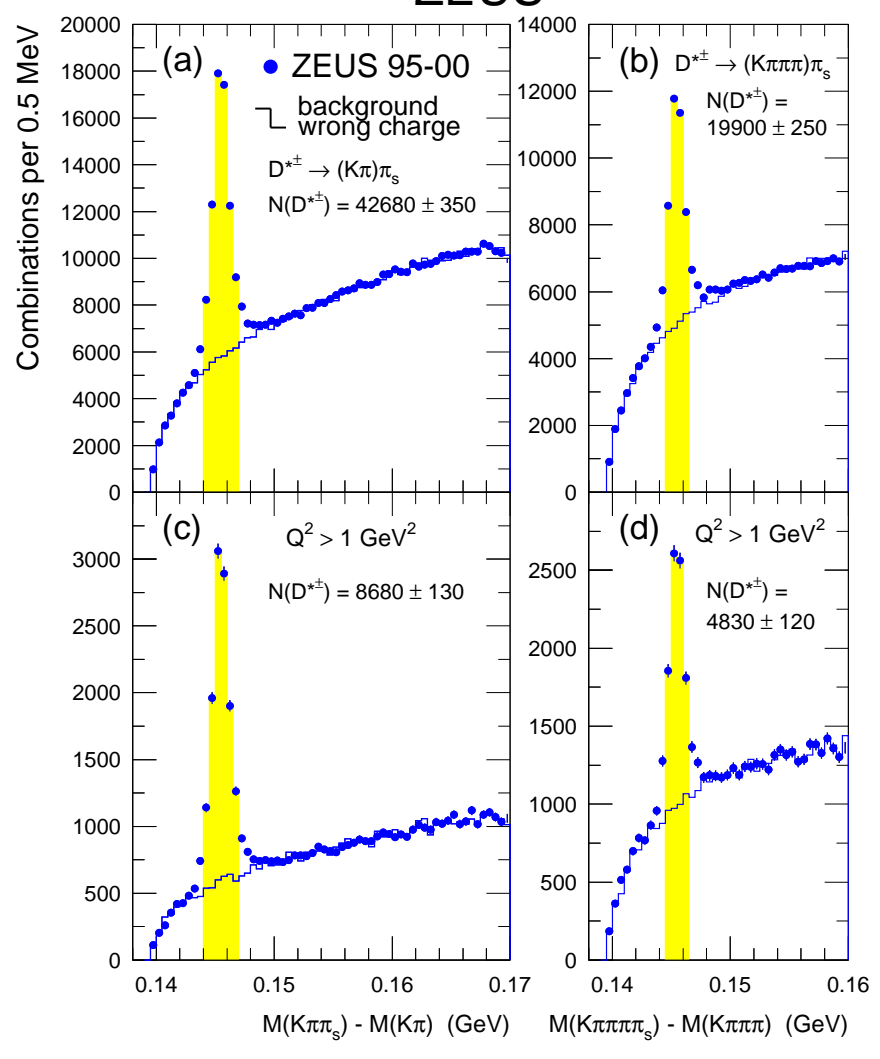

two $D^{*}$ decay channels:

$$
\begin{aligned}
& p_{T}\left(D^{*}\right)>1.35 \mathrm{GeV} \text { for } D^{*} \rightarrow(K \pi) \pi_{s} \\
& p_{T}\left(D^{*}\right)>2.8 \mathrm{GeV} \text { for } D^{*} \rightarrow(K \pi \pi \pi) \pi_{s} \\
& \left|\eta\left(D^{*}\right)\right|<1.6 \text { for both channels }
\end{aligned}
$$

$$
\begin{aligned}
& \text { Yellow bandis used } \\
& \text { for } \Theta_{c}^{0} \text { search: } \\
& \boldsymbol{N}\left(D^{*}\right) \sim \mathbf{6 2 5 0 0} \text {, full sample } \\
& \boldsymbol{N}\left(D^{*}\right) \sim \mathbf{1 3 5 0 0}, Q^{2}>\mathbf{1} \mathrm{GeV}^{\mathbf{2}}
\end{aligned}
$$




\section{$M\left(D^{*} p\right)$ in DIS and photoproduction}

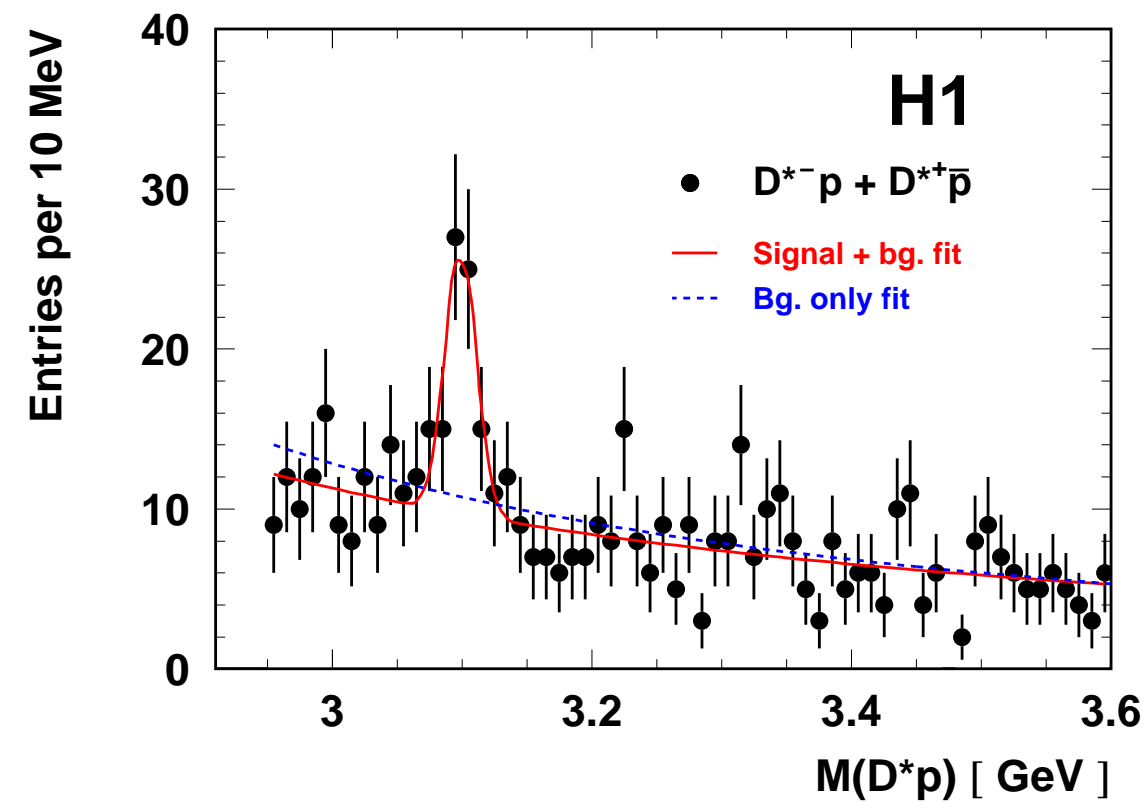

Clean signal in DIS

(in both $D^{*+} \bar{p}$ and $D^{*-} p$ )

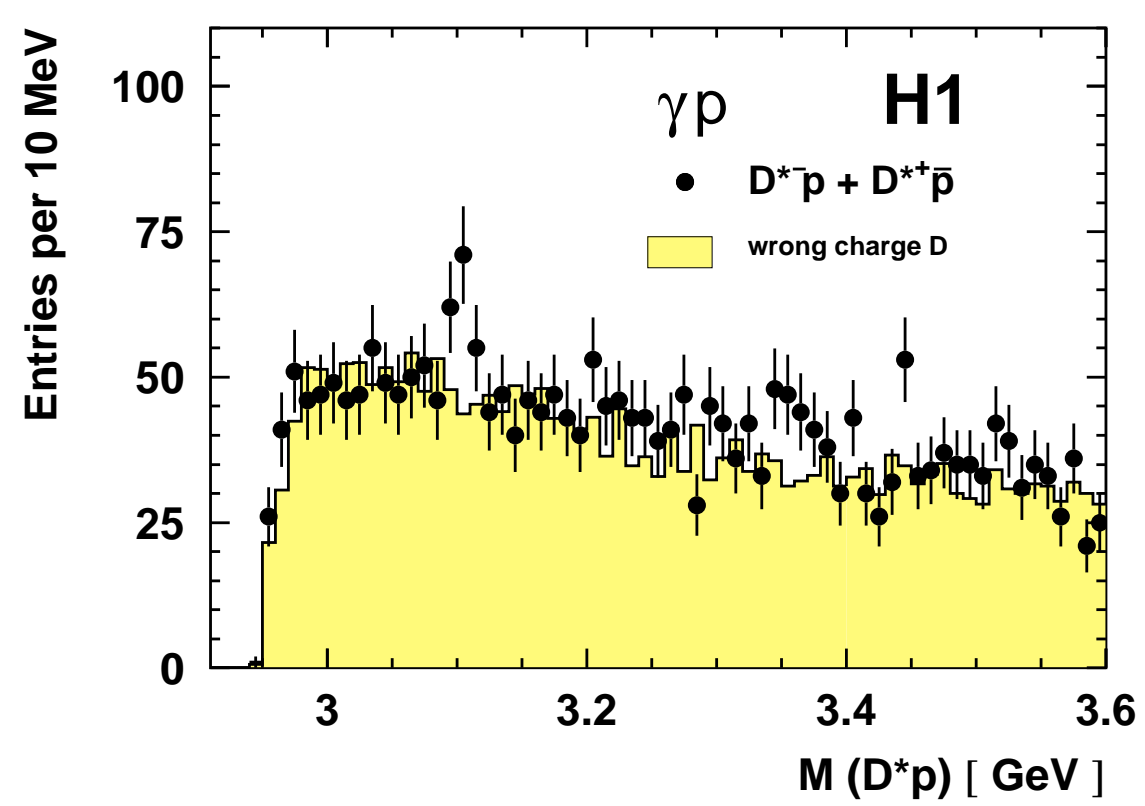

Signal for $Q^{2}<1 \mathrm{GeV}^{2}$ at the same mass

Fit Gaussian + background (2 par.):

$$
\begin{aligned}
& N\left(\Theta_{c}^{0}\right)=50.6 \pm 11.2 \\
& M\left(\Theta_{c}^{0}\right)=3099 \pm 3(\text { stat. }) \pm 5(\text { syst. }) \mathrm{MeV} \\
& \sigma\left(\Theta_{c}^{0}\right)=12 \pm 3 \mathrm{MeV} \text { (consist. with resolution) }
\end{aligned}
$$

visible rate $R\left(\Theta_{c}^{0} \rightarrow D^{*} p / D^{*}\right)=1.46 \pm 0.32 \%$ (prel.) or "roughly $1 \%$ " 


\section{$M\left(D^{*} p\right)$ for large proton momenta}
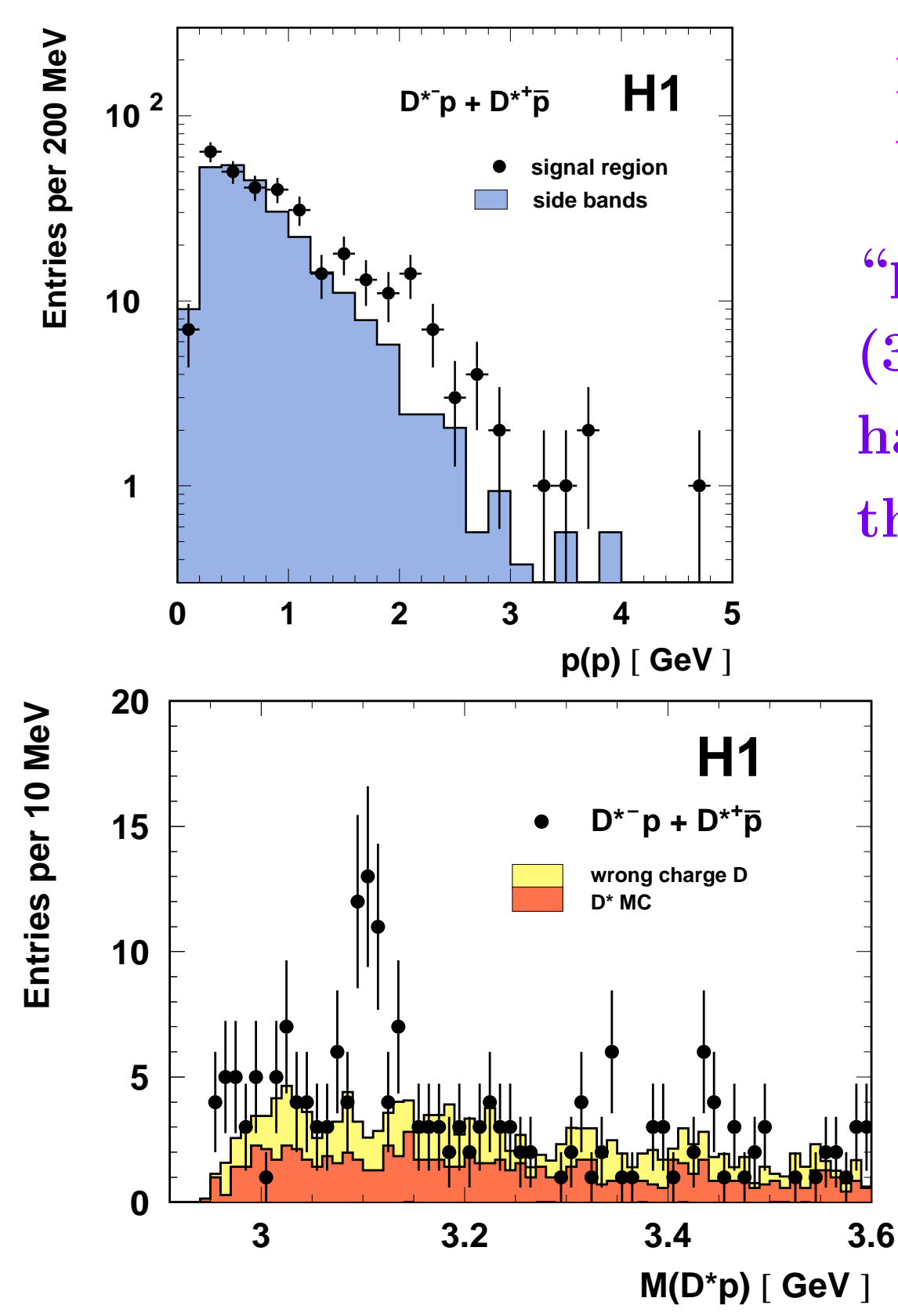

particles taken as protons w/o $d E / d x$ requirements

"protons" from signal region $\left(3.085<M\left(D^{*} p\right)<3.115 \mathrm{GeV}\right)$ have harder momentum distribution than "protons" from side bands

For $P(p)>2 \mathrm{GeV}$, clean signal is seen even $\mathrm{w} / \mathrm{o}$ use of $d E / d x$

$\Longleftarrow$ background is well described by 2 -component model 


\section{$p(\bar{p})$ identification, ZEUS}

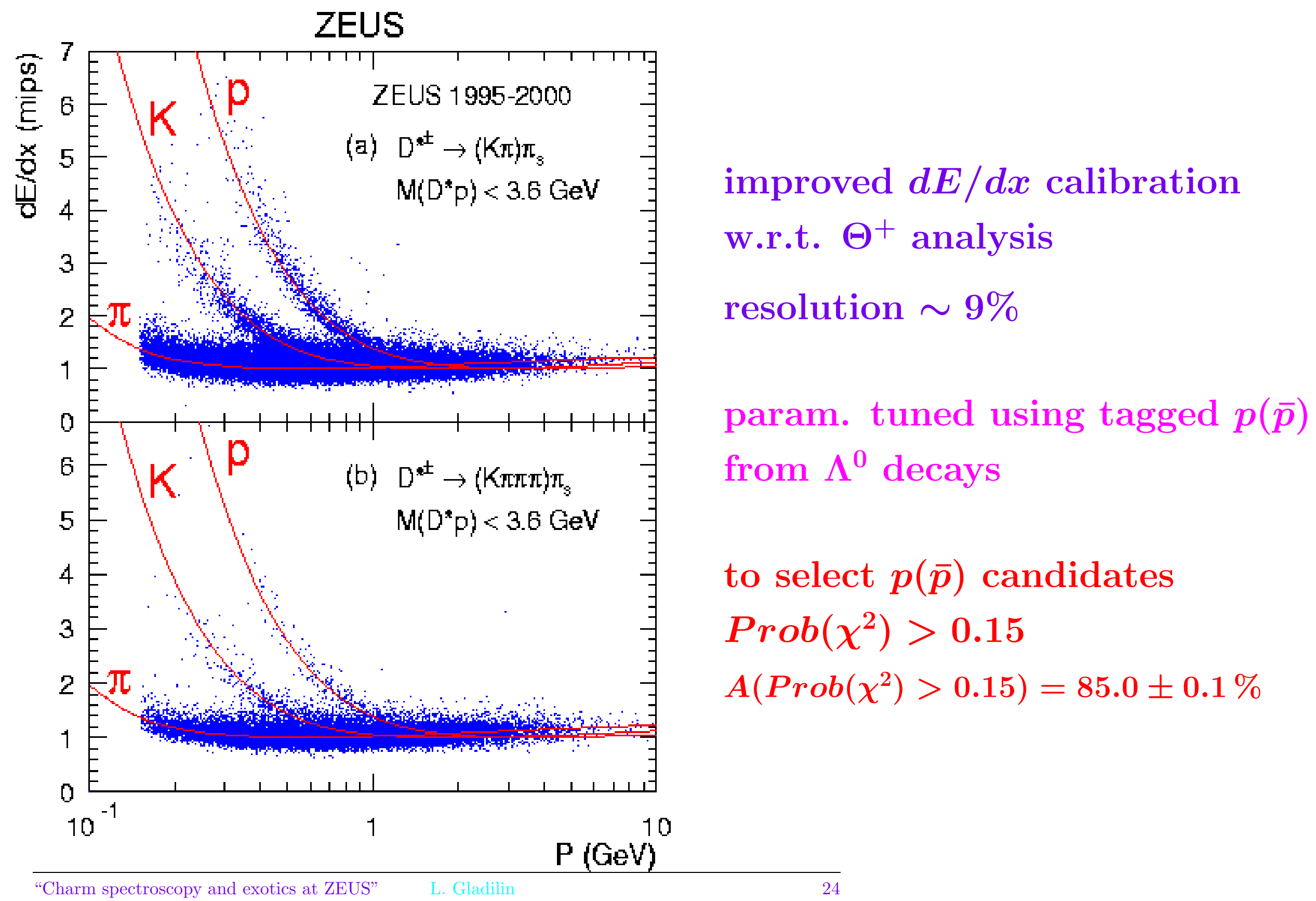




\section{$M\left(D^{*} p\right)$, ZEUS}

ZEUS

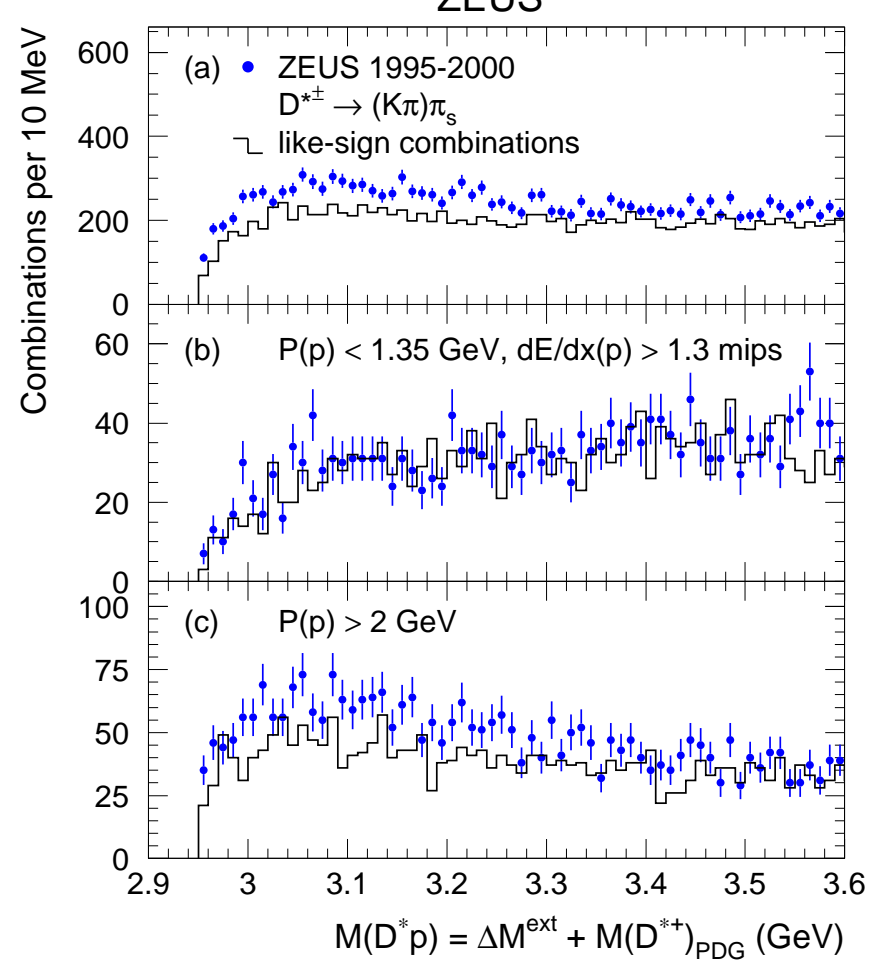

\section{ZEUS}

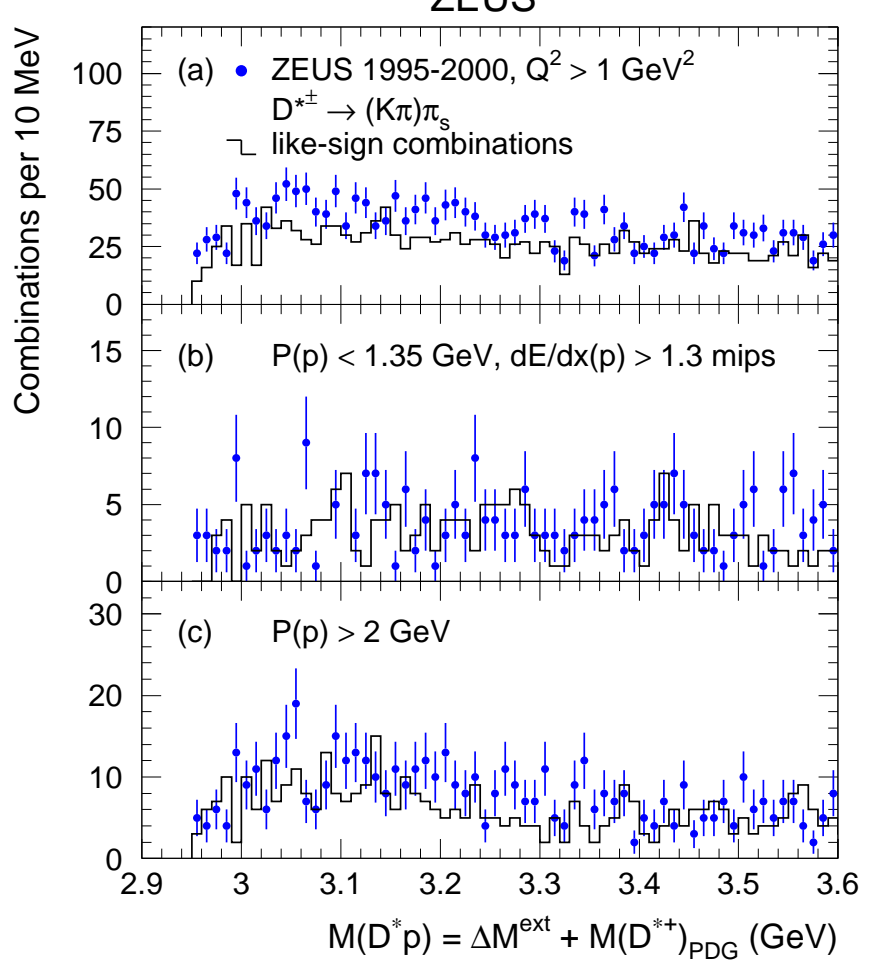

ZEUS

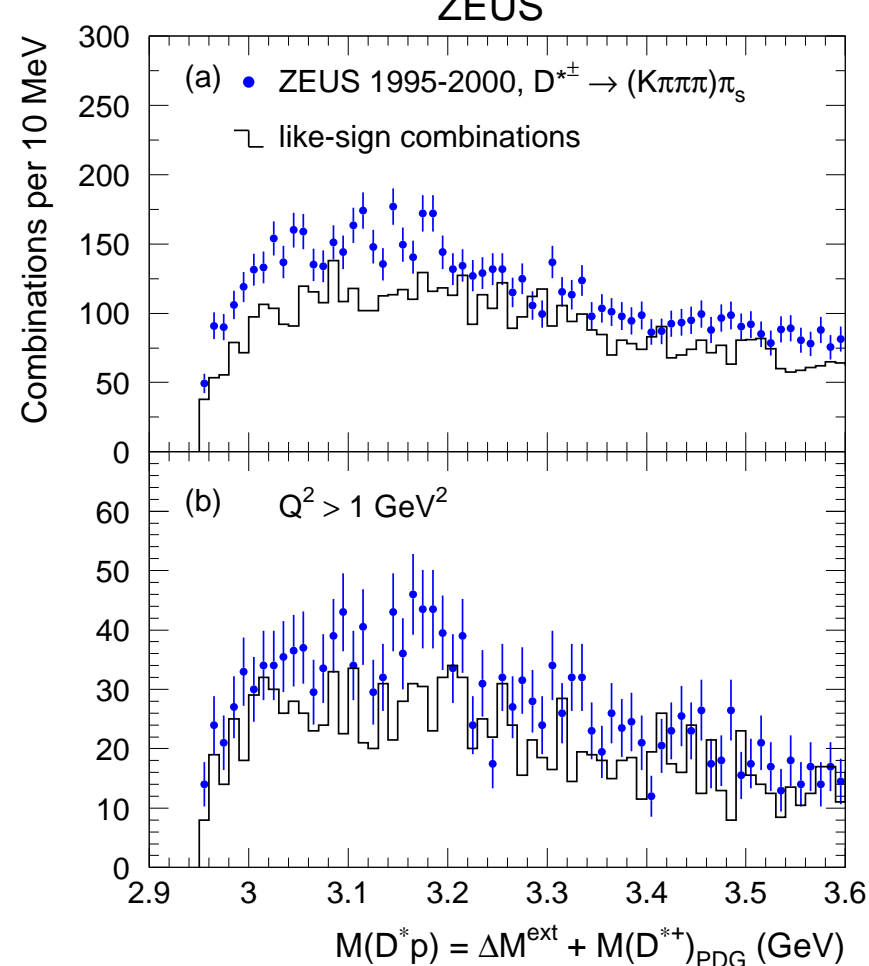

\section{no signal in either distribution}




\section{ZEUS $\Theta_{c}^{0}$ MC and extended $\Delta M$ method}

To prepare signal $\mathrm{MC}, \Theta_{c}^{0}$ was emulated by redefining mass, width and decay channel of $\Sigma_{c}^{0}(d d c)$
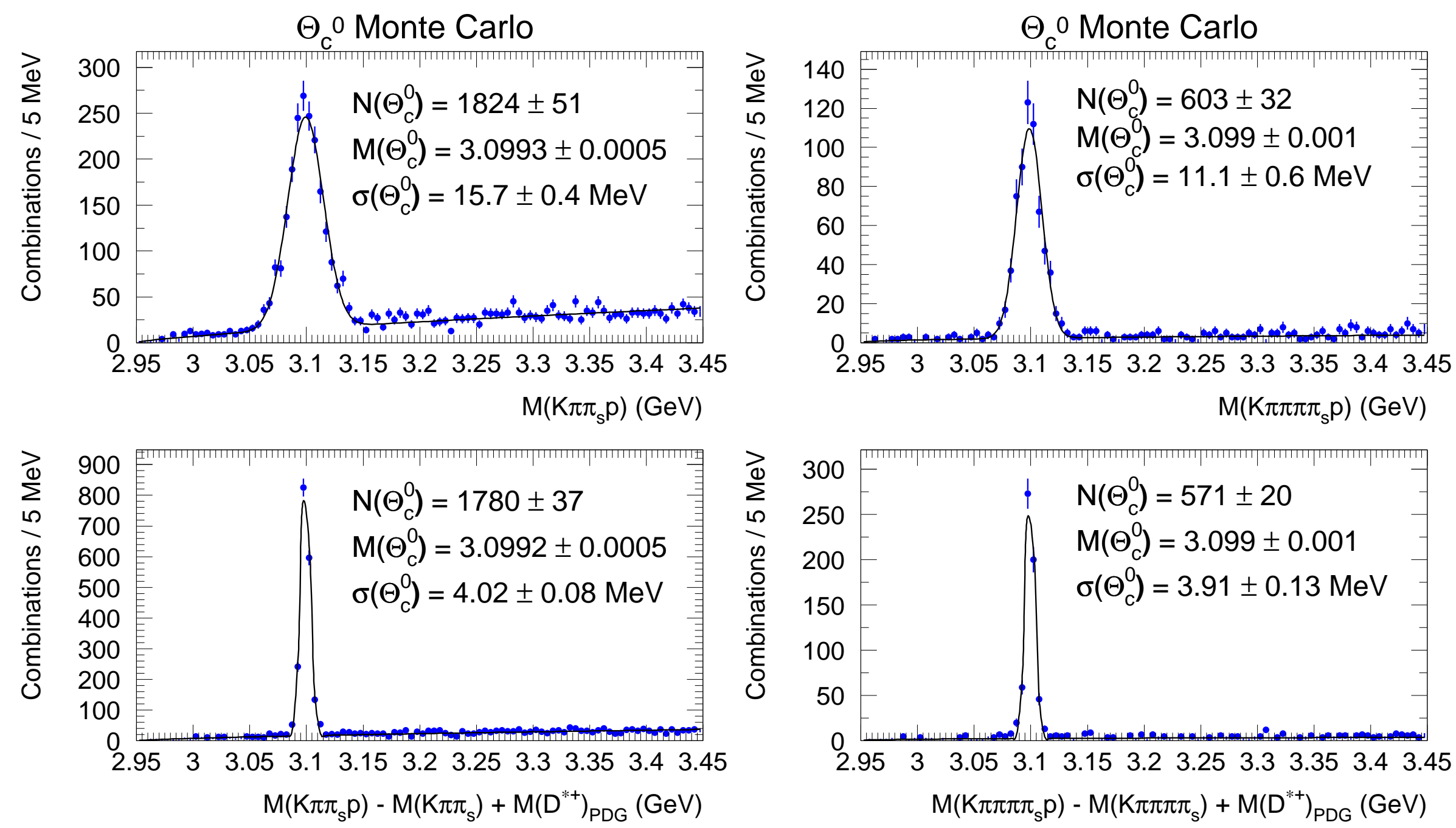

resolution is $\sim 4 \mathrm{MeV}$ (w.r.t. $\sim 7 \mathrm{MeV}$ in $\mathrm{H1}$ analysis) 


\section{Orbitally excited $\mathrm{P}$-wave $\boldsymbol{D}$ mesons}

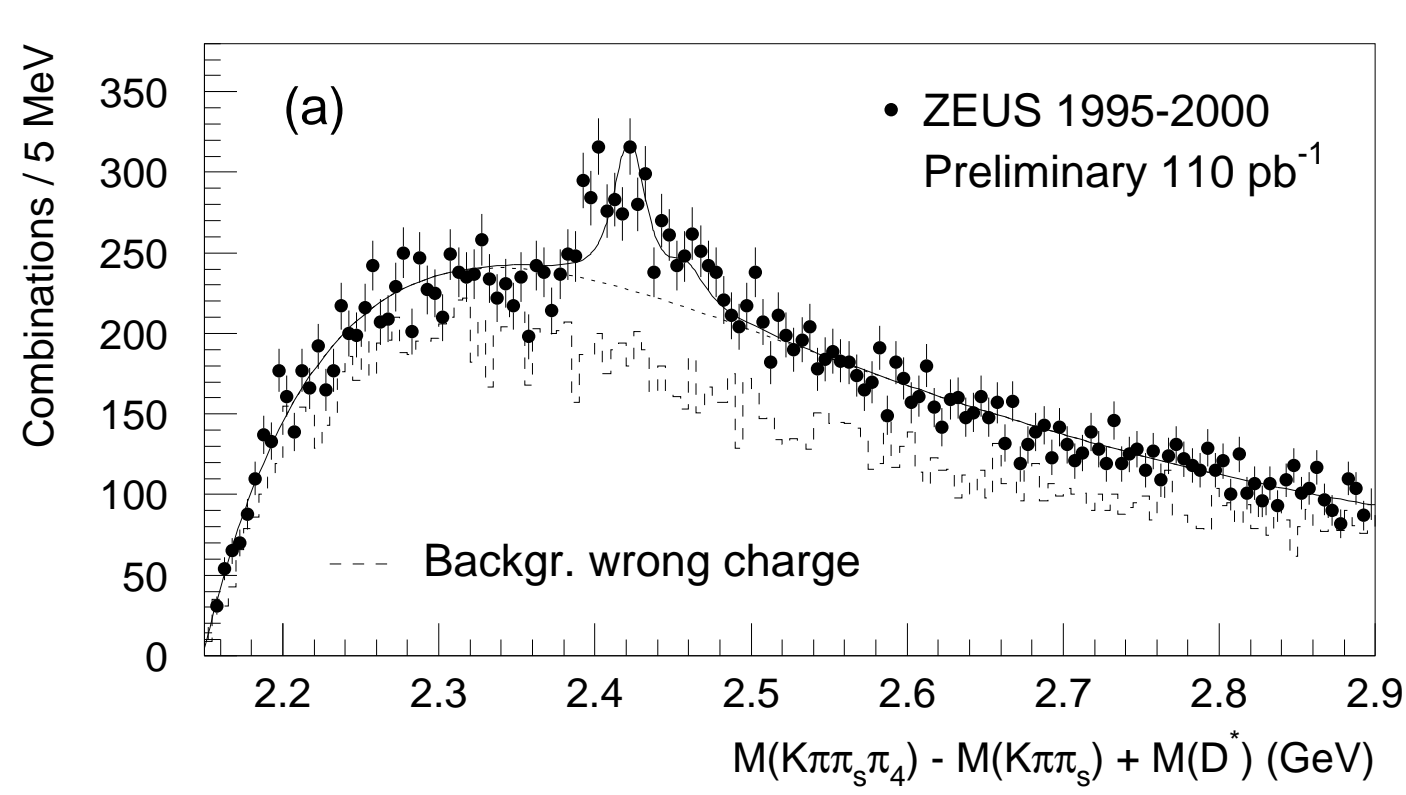

$$
\frac{D_{1}^{0}(2420), D_{2}^{* 0}(2460) \rightarrow D^{* \pm} \pi^{\mp}}{\Delta M^{e x t}=M\left(K \pi \pi_{S} \pi_{4}\right)-M\left(K \pi \pi_{s}\right)}
$$

2-dimensional fit with fixed $M, \Gamma$, resolution and helicity distr. :

$$
\begin{array}{ll}
\frac{d N}{d \cos \alpha} \propto 1+3 \cos ^{2} \alpha & \left(1^{+}, L+s=3 / 2\right) \\
\frac{d N}{d \cos \alpha} \propto 1-\cos ^{2} \alpha & \left(2^{+}, L+s=3 / 2\right)
\end{array}
$$

helicity angle $\alpha$ : between $\pi_{4}$ and $\pi_{s}$

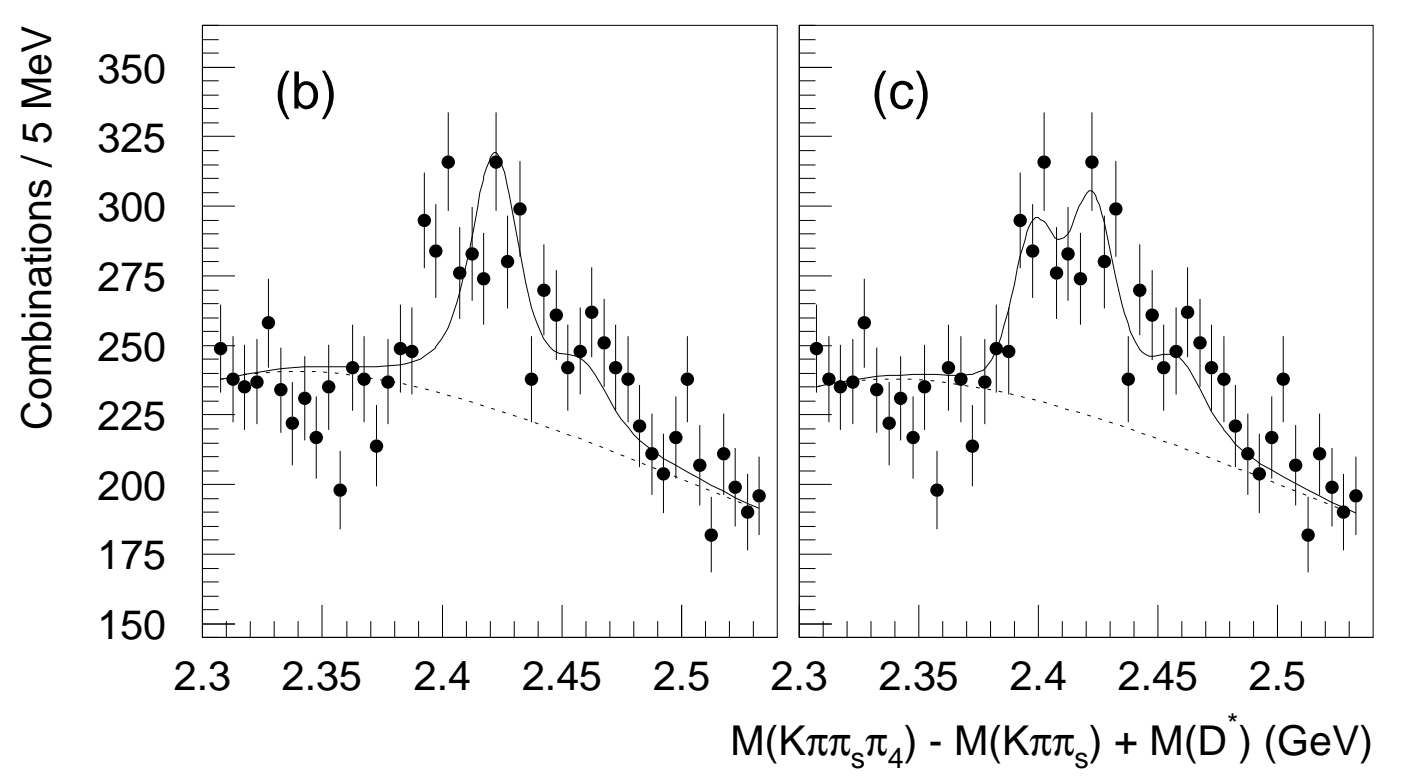
in $D^{* \pm}$ rest frame

$N\left(D_{1}^{0}\right)=526 \pm 65$

$N\left(D_{2}^{* 0}\right)=203 \pm 60$

Additional narrow bump ?

$N=211 \pm 49$

$M=2398.1 \pm 2.1$ (stat.) ${ }_{-0.8}^{+1.6}$ (syst.) $\mathrm{MeV}$

New $D$ meson ? Interference? 


\section{Search for radially excited $D^{* \prime \pm}$ meson}

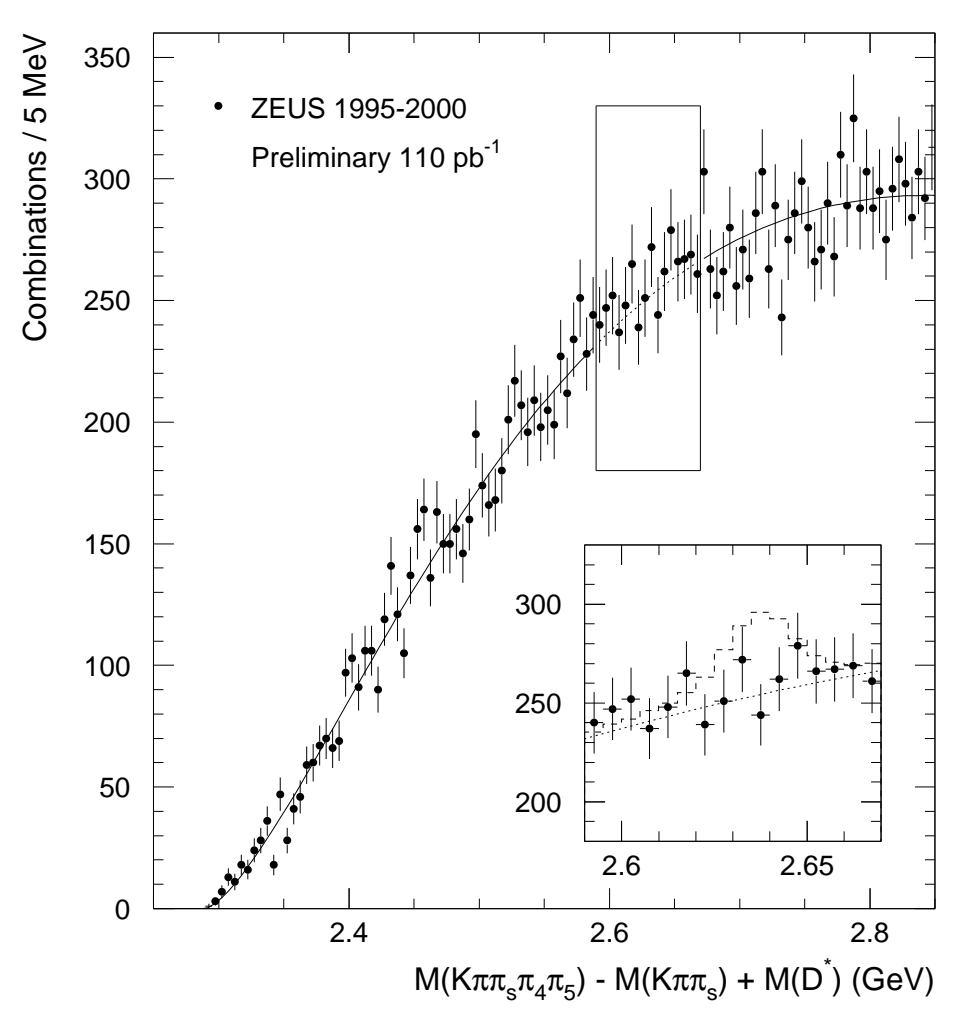

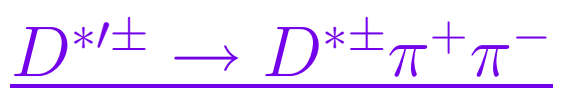

Observed by DELPHI $(\sim 5 \sigma): M=2637 \mathrm{MeV}$

$\Gamma<15 \mathrm{MeV}$

CLEO and OPAL did not confirm

$\Longleftarrow$ ZEUS search

$\Delta M^{e x t}=M\left(K \pi \pi_{S} \pi_{4} \pi_{5}\right)-M\left(K \pi \pi_{s}\right)$

Search window: $2.59<\Delta M^{e x t}+M\left(D^{*+}\right)<2.67 \mathrm{GeV}$ covers both predictions and DELPHI's observation after backgr. subtraction: " $N\left(D^{* 1 \pm}\right)$ " $=91 \pm 75$

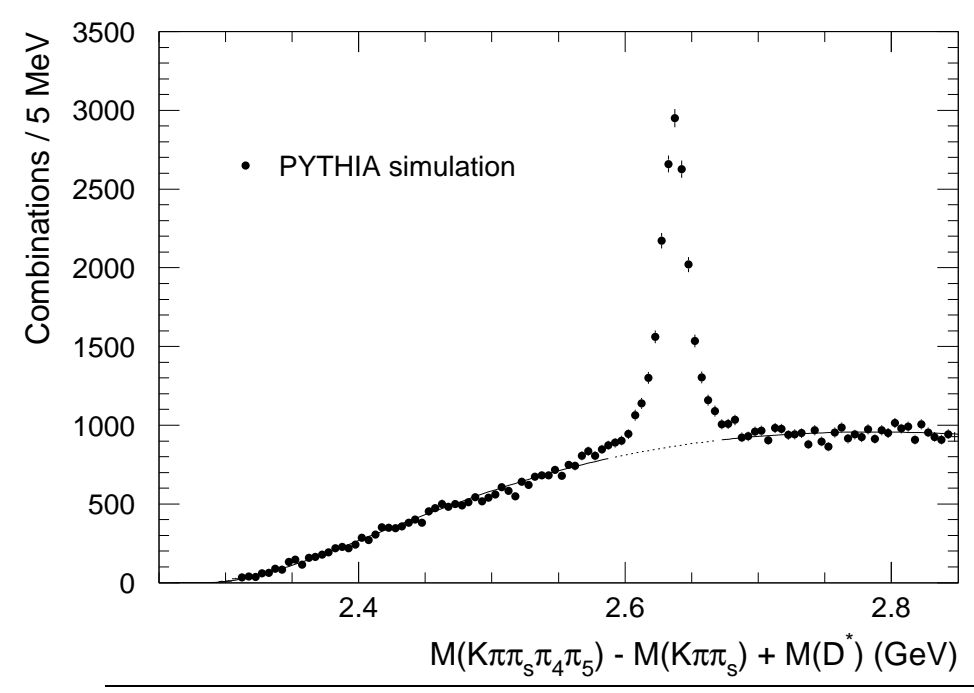

Using world average for $f\left(c \rightarrow D^{*+}\right)$ :

$f\left(c \rightarrow D^{* /+}\right) \cdot B_{D^{*++} \rightarrow D^{*+} \pi^{+} \pi^{-}}<0.7 \% \quad(95 \% \quad$ C.L. $)$ (ZEUS prel.)

somewhat stronger than the $0.9 \%$ limit obtained by OPAL 